\title{
TIME-DELAYED INSTABILITIES IN COMPLEX BURGERS EQUATIONS
}

\author{
MARTA STRANI AND BENJAMIN TEXIER
}

\begin{abstract}
For Burgers equations with real data and complex forcing terms, Lerner, Morimoto and $\mathrm{Xu}$ [Instability of the Cauchy-Kovalevskaya solution for a class of non-linear systems, Amer. J. Math. 2010] proved that only analytical data generate local $C^{2}$ solutions. The corresponding instabilities are however not observed numerically; rather, numerical simulations show an exponential growth only after a delay in time. We argue that numerical diffusion is responsible for this time delay, as we prove that for Burgers equations in the torus with small viscosity and a complex forcing, oscillating data generate solutions which grow linearly in time before growing exponentially. Numerical simulations illustrate the results.
\end{abstract}

\section{INTRODUCTION}

For one-dimensional Burgers equations with small viscosity, and a complex forcing term:

$$
\text { (B) } \partial_{\varepsilon} \quad \partial_{t} u+u \partial_{x} u-\varepsilon \partial_{x}^{2} u=i
$$

where $u(\varepsilon, t, x) \in \mathbb{C}$ depends on time $t \in \mathbb{R}_{+}$, space $x \in \mathbb{T}=\mathbb{R} / \mathbb{Z}$, and the small parameter $\varepsilon>0$, we prove that oscillating data generate solutions that grow linearly in time before growing exponentially. In particular, these solutions behave qualitatively very much like solutions to the viscous degenerate Cauchy-Riemann equation

$$
\partial_{t} v+i t \partial_{x} v-\varepsilon \partial_{x}^{2} v=0
$$

for which the instability (of the Cauchy problem, for real data) is manifest only after a delay in time.

This sheds new light on the strong instability result of Lerner, Morimoto, and $\mathrm{Xu}$ [3] for equation $(\mathrm{B})_{0}$, corresponding to the inviscid case $\varepsilon=0$.

1.1. Background. In the inviscid case $\varepsilon=0$, Lerner, Morimoto and Xu proved [3] that if a real datum generates a local $C^{2}$ solution of the inviscid equation $(\mathrm{B})_{0}$, then the datum must be analytic. This reveals a strong instability of the Cauchy problem for $(\mathrm{B})_{0}$.

It is important to note the degeneracy in $(\mathrm{B})_{0}$ : given a real datum $a$, the linearized first-order operator $\partial_{t}+a \partial_{x}$ is hyperbolic at $t=0$; for arbitrarily small $t>0$, however, due to the complex forcing, if a solution $u(t)$ exists then presumably $\Im m u(t) \neq 0$, so that the linearized operator is not hyperbolic. The degeneracy is apparent with the change of unknown $u=i t+v$, by which $(\mathrm{B})_{0}$ becomes equation 
$\partial_{t} v+(i t+v) \partial_{x} v=0$. Linearizing at $v=0$, we obtain the degenerate Cauchy-Riemann equation

$$
\partial_{t} w_{1}+i t \partial_{x} w_{1}=0
$$

Of course, the Cauchy problem for $(1.2)$ is ill-posed in Sobolev spaces. The instability, however, is slower to develop than for the (non-degenerate) Cauchy-Riemann equation

$$
\partial_{t} w_{2}+i \partial_{x} w_{2}=0
$$

Indeed, a datum $a(x)$ will generate solutions $w_{1}$ to 1.2 and $w_{2}$ to 1.3 which satisfy

$$
\left|\hat{w}_{1}(t, \xi)\right|=|\hat{a}(\xi)| e^{t^{2} \xi / 2}, \quad\left|\hat{w}_{2}(t, \xi)\right|=|\hat{a}(\xi)| e^{t \xi},
$$

so that, for a given frequency $\xi$ and small $t$, there holds $\left|\hat{w}_{1}(t, \xi)\right| \ll\left|\hat{w}_{2}(t, \xi)\right|$.

This degeneracy is inherent to the phenomenon of transition from hyperbolicity (at $t=0$ for $(\mathrm{B})_{0}$ ) to ellipticity (at $t>0$ for $(\mathrm{B})_{0}$ ).

The article [4] expounds on [3] by systematically describing the transition to ellipticity (defined as loss of hyperbolicity) for first-order systems. It is shown in [4] that a loss of hyperbolicity implies a strong Hadamard instability, that is an instantaneous deviation estimate: some nearby data (as measured in a strong norm) may generate solutions which are instantly driven apart (as measured in a weak norm). We write "may generate solutions", conditional, since in this setting the existence of solutions typically cannot be proved ${ }^{1}$. Of course if some Sobolev data do not generate solutions at all, then the Cauchy problem is even more strongly ill-posed (absence of a solution operator, compared to absence of Hölder estimates for a solution operator).

Here we are interested in the effect of adding a small viscous term to an equation and a solution that experience a transition from hyperbolicity to ellipticity, as we focus on $(\mathrm{B})_{\varepsilon}$, particularly in relation to 1.1 .

1.2. Observations. Our starting observation is that instantaneous instabilities for $(B)_{0}$ are not observed numerically. Precisely, our numerical tests show for small times a linear growth of the imaginary part of the numerical solution instead of a catastropic amplification.

Our second observation is that this behavior, linear growth in time of the imaginary part for small times, is precisely the one recorded for the viscous degenerate Cauchy-Riemann equation (1.1). Indeed, for the solution $v$ to 1.1 issued from $v(\varepsilon, 0, x)=a(\varepsilon, x)$, there holds, by Fourier transform and direct time integration,

$$
|\hat{v}(\varepsilon, t, k)|=|\hat{a}(\varepsilon, k)| \exp \left(2 \pi t k\left(\frac{t}{2}-2 \pi \varepsilon k\right)\right), \quad k \in \mathbb{Z},
$$

\footnotetext{
${ }^{1}$ Unless analyticity, of the data (in $x$ ) and the differential operator (in $u$ ), is assumed. For longtime Cauchy-Kovalevskaya results which prove existence together with Hadamard ill-posedness, see Métivier [6] in the non-degenerate case of an elliptic Cauchy problem, and Morisse [8] in the degenerate case of a transition to ellipticity.
} 
so that $\hat{v}(\cdot, k)$ grows exponentially only for $t \geq 4 \pi \varepsilon k$, and only if $k$ belongs to the support of $\hat{a}$.

For instance, if the initial datum is highly oscillating: $a(x)=\sin (2 \pi x / \varepsilon)$, then the amplification occurs only after $t=4 \pi$. And if the smallest positive frequency in the datum is $k_{0} \in \mathbb{Z}_{+}^{*}$, as in $a(x / \varepsilon)=\sin \left(2 \pi k_{0} x / \varepsilon\right)$, then the amplification for (1.1) occurs only after $t=4 \pi k_{0}$.

We define the transition time as the smallest positive time for which the symbol has negative real part. We compute the symbol associated with equation (1.1) by changing $\partial_{x}$ into $2 \pi i \xi$ (Fourier transform), so that the symbol here is

$$
i t(2 \pi i \xi)-\varepsilon(2 \pi i \xi)^{2}=-(2 \pi \xi)(t-2 \pi \varepsilon \xi) .
$$

Real negative values occur for $t \geq 2 \pi \varepsilon \xi$. For instance, for the datum $a(\varepsilon, x)=$ $\sin \left(2 \pi x k_{0}\right)$, with $k_{0} \in \mathbb{Z}^{*}$, we consider frequencies $\xi \in k_{0} \mathbb{Z}$, and the smallest $t$ for which the symbol has real negative eigenvalues is $2 \pi \varepsilon k_{0}$. This is the transition time (for this equation and this datum). For the datum $a(\varepsilon, x)=\sin \left(2 \pi k_{0} x / \varepsilon\right)$, the transition time is $2 \pi k_{0}$.

By amplification time we mean smallest possible time for which an adequate measure of the solution is greater than the same measure of the datum. For instance, in the case of datum $a(\varepsilon, x)=\sin \left(2 \pi k_{0} x / \varepsilon\right)$, the representation (1.4) for the solution $v$ to (1.4) is non-trivial only if $k \in k_{0} \mathbb{Z} / \varepsilon$. In particular, the smallest possible $t$ for which $|\hat{v}(\varepsilon, t, k)| \geq|\hat{a}(\varepsilon, k)|$ for some $k$ is $t=4 \pi k_{0}$, corresponding to $k=k_{0} / \varepsilon$. The amplification time is $4 \pi k_{0}$, greater than the transition time.

In particular, the amplification occurs only after the transition. This is particularly meaningful in view of our results stated in Section 1.3 below.

Based on these observations, our guess was that numerical diffusion was responsible for the defect in instantaneous amplification in the simulations. By numerical diffusion, we mean the fact that the standard Lax-Friedrichs scheme that we used for the simulation of $(\mathrm{B})_{0}$, with time step $\sigma$ and length interval $h$, is consistent at order 1 with $(\mathrm{B})_{0}$, but consistent at order 2 with $(\mathrm{B})_{\varepsilon}$ with $\varepsilon=h^{2} /(2 \sigma)$.

The analogy with (1.1) then suggested that exponential amplification for the numerical solutions to $(\mathrm{B})_{0}$ would occur for ulterior times. This was confirmed by numerical simulations on longer time intervals.

Numerical solutions to $(B)_{\varepsilon}$ are pictured on Figure 1. We show tests for the initial data $a(x)=\sin (N \cdot 2 \pi x)$, with $N \in\{8,16,24\}$. We recorded the maximum value over the spatial grid of the imaginary parts of the numerical solutions every 20 time steps. The thick lines are the graphs of these maxima as functions of time. The solution that seems to blow up around $t=.2$ corresponds to $N=8$. The one that seems to blow up around $t=.45$ corresponds to $N=16$. The rightmost one, seemingly blowing up around $t=.7$, corresponds to $N=24$. By comparison, we picture on the same graph the corresponding "linearized" solutions, in thin lines. These solutions are translates of solutions to (1.1). Indeed, if $u$ solves $(\mathrm{B})_{\varepsilon}$, then $v:=u-i t$ solves $\partial_{t}+(i t+v) \partial_{x} v-\varepsilon \partial_{x}^{2} v=0$, and linearizing at $v=0$ we find (1.1). 


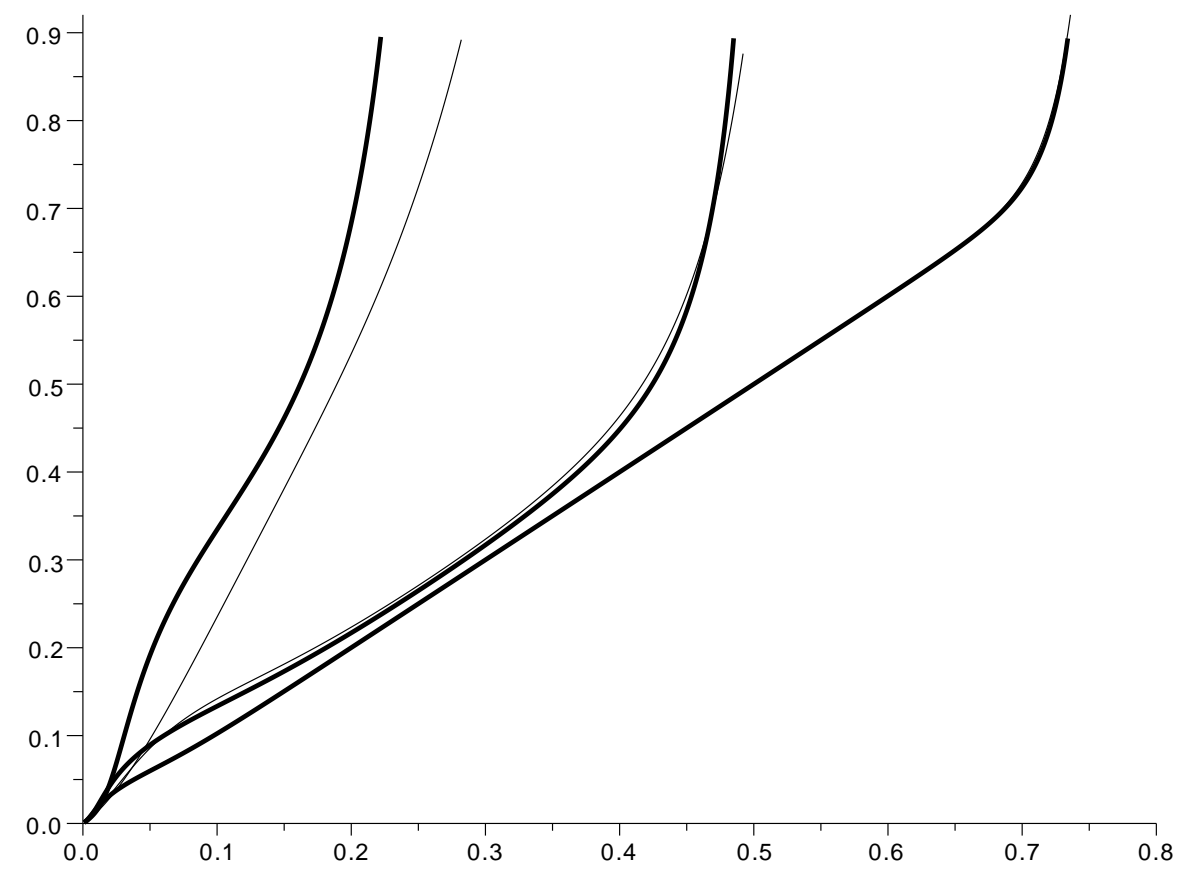

Figure 1. Numerical and "linearized" solutions for $N=8,16$ and 24 .

The solution to (1.1) issued from $a(x)=\sin (N \cdot 2 \pi x)$ is

$$
v(\varepsilon, t, x)=\frac{1}{2 i} e^{t^{2} N / 2-\varepsilon t N^{2}} e^{2 i \pi N x}-\frac{1}{2 i} e^{-t^{2} N / 2-\varepsilon t N^{2}} e^{-2 i \pi N x},
$$

so that

$$
\max _{x \in \mathbb{T}} \Im m v(\varepsilon, t, x)=\frac{1}{2}\left(e^{t^{2} N / 2-\varepsilon t N^{2}}-e^{-t^{2} N / 2-\varepsilon t N^{2}}\right) .
$$

The thin lines on Figure 1 are the graphs of $t \rightarrow t+\max _{\mathbb{T}} \Im m v(\varepsilon, t, \cdot)$, with $v$ as above. For $N=16$ we find good agreement between the numerical and the linearized solution. For $N=24$, on the scales of the picture the graphs are essentially indistinguishable. This indicates that the approximation of $(B)_{\varepsilon}$ by $(1.1)$ seems quite accurate for oscillating data, even if the amplitude of the data is $O(1)$.

Here the ratio $\varepsilon=h^{2} /(2 \sigma)$ is set equal to $2.5 \cdot 10^{-3}$, with the spatial step $h=$ $5 \cdot 10^{-4}$ and the temporal step $\sigma=5 \cdot 10^{-5}$.

On Figure 1, the rightmost graph, corresponding to $N=24$, shows a linear behavior in time, followed by a quick amplification. The computing time for this simulation is about 250 minutes on a laptop computer. The amplification is apparent only about $t=.7$, well after 150 minutes of computing time. 


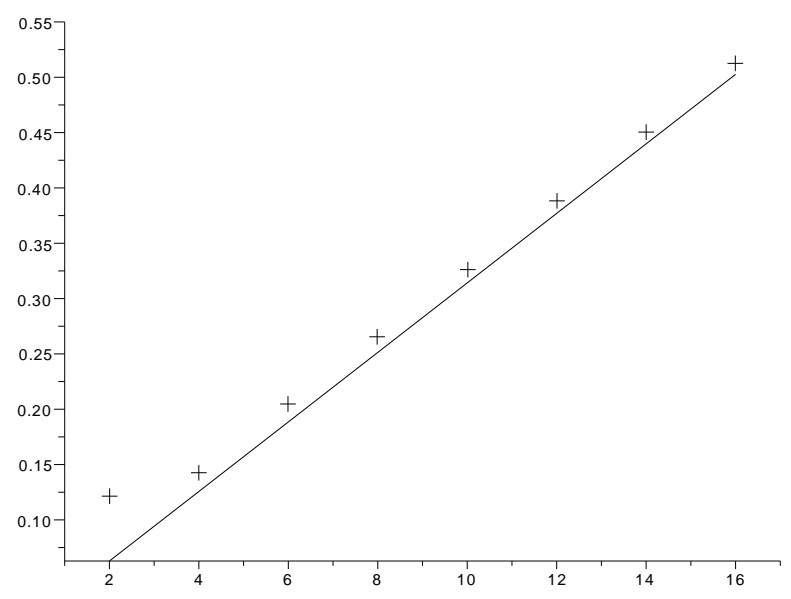

Figure 2. Amplification times for different values of the initial frequency.

Next we examined how the amplification time varied according to the initial oscillations and the viscosity. According to (1.4), the amplification time for equation (1.1) is $t_{\mathrm{cc}}=4 \pi \varepsilon N$, where $N$ is the smallest non-zero mode in the real initial datum. In particular, it has a linear dependence both in $\varepsilon$ and in the smallest mode in the datum. We compared this linear amplification time with $t_{\mathrm{f}}$, the final computing time in our simulations, defined as follows.

In the main time-loop of our numerical scheme, we imposed the CFL condition

$$
\frac{\sigma}{h}=0.1<\frac{0.4}{\max \left(\max _{j}\left|\Re e u^{n}(j)\right|, \max \left|\Im m u^{n}(j)\right|\right)},
$$

where $u^{n}$ represents the numerical solution to $(\mathrm{B})_{\varepsilon}$ at step $n$. In particular, the simulations stopped when condition (1.5) broke down, corresponding to the smallest positive time for which the $L^{\infty}$ norm of the discrete solution is greater or equal to 4. The final computing time $t_{\mathrm{f}}$ is the largest computing time before (1.5) breaks down.

On Figure 2 we compare $t_{\mathrm{cc}}=4 \pi \varepsilon N$ (drawn as full line) with $t_{\mathrm{f}}$ (crosses), for the initial data $a(x)=\sin (N \cdot 2 \pi x)$, for $N$ taking even values from 2 to 16 . The values of $h$ and $\sigma$, hence $\varepsilon$, are as in Figure 1. We see that for $N$ not too small, there is good agreement between $t_{\mathrm{cc}}$ and $t_{\mathrm{f}}$. It is not surprising to find $t_{\mathrm{f}}$ to be greater than $t_{\mathrm{cc}}$, since when the maximum of the numerical solution equals 4 , the amplification has already started for some time. On Figure1, the graphs are truncated before the final computing time for better legibility.

On Figure 3, we show tests for the dependence of the amplification time on the smallest non-zero mode in the datum. We compare $t_{\mathrm{cc}}$ (full line) with $t_{\mathrm{f}}$ (crosses), for the same values of $h$ and $\sigma$ as above, and for 8 different initial data, of the form 


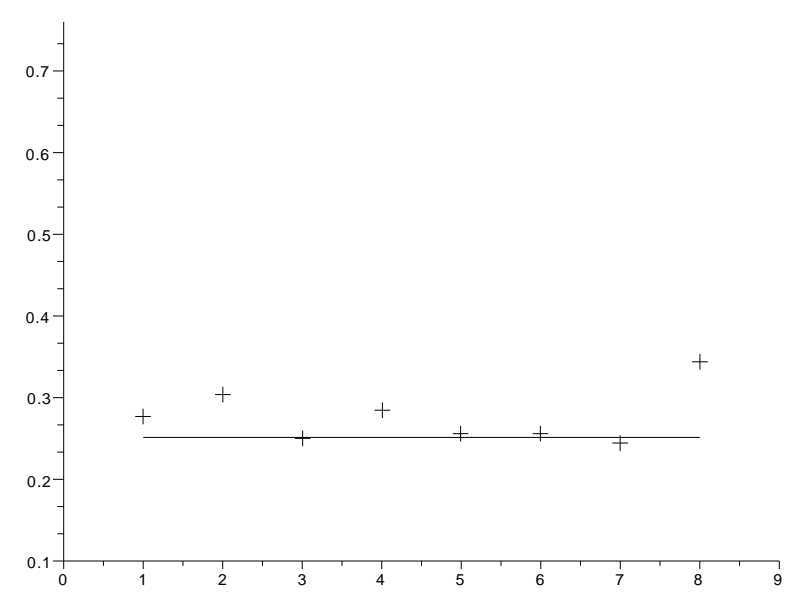

Figure 3. Amplification times for different initial data.

$\sum_{1 \leq j \leq 3} a_{j} \sin \left(N_{j} \cdot 2 \pi x\right)$, with specific values as follows, the $x$-coordinate on Figure 3 corresponding to the case number:

- case $1:\left(N_{1}, N_{2}, N_{3}\right)=(4,0,0),\left(a_{1}, a_{2}, a_{3}\right)=(1,0,0)$; this is a test case with only one initial oscillation;

- case i, with $2 \leq i \leq 5:\left(N_{1}, N_{2}, N_{3}\right)=(4,4+2(i-1), 0),\left(a_{1}, a_{2}, a_{3}\right) \equiv$ $(1,1,0)$;

- cases 6,7,8: $\left(N_{1}, N_{2}, N_{3}\right) \equiv(4,6,8)$, with coefficients equal to $(1,1,1)$ then $(1,2,1)$ and finally $(1,2,-1)$.

The straight line is the linear amplification time $t_{\mathrm{cc}}=4 \cdot 4 \pi \varepsilon$, common to all cases. We observe that the numerical values of $t_{\mathrm{f}}$ are relatively close to the linear value. Of course the scale on the $y$-axis here plays a crucial role in conveying the impression that the approximation by (1.1) makes sense. The 8 cases tested on Figures 3 involve oscillations $\sin (2 \pi N x)$, with $N$ ranging from 4 to 12 . Therefore we chose extremal values on the $y$-axis corresponding to the extremal amplification times associated with these frequencies, that is values ranging from $t_{\mathrm{cc}}(N=4)=.251$ to $t_{\mathrm{cc}}(N=12)=.754$.

We finally tested the dependence of the final computing time on the viscosity. The results are pictured on Figure 4, with $t_{\mathrm{cc}}=4 \pi \varepsilon N$ drawn as a full line, and $t_{\mathrm{f}}$ as crosses. Here the datum is $a(x)=\sin (10 \cdot 2 \pi x)$. We tested values of the number $J$ of points on the spatial grid, from $J=2000$ to $J=600$, with increments of 200 . The associated spatial step is $h=1 / J$. We fixed $\sigma=h / 10$, so that $\varepsilon=h^{2} /(2 \sigma)=5 / J$. The corresponding values of $\varepsilon$ range from $2.5 \cdot 10^{-3}$ (for $J=2000$ ) to $8.33 \cdot 10^{-3}$ (for $J=600$ ). There is an excellent agreement with the linear approximation. 


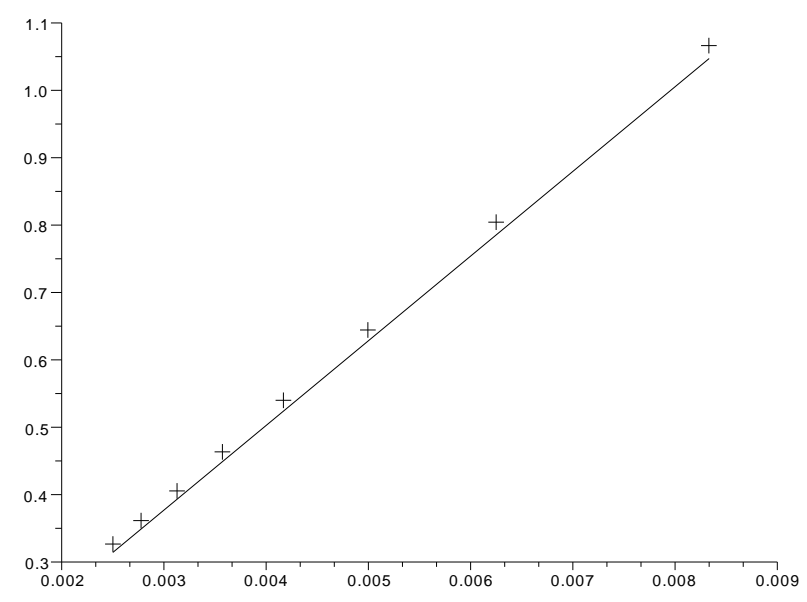

Figure 4. Amplification times depending on viscosity.

1.3. Results. In a first step (Section 2), we show by elementary a priori bounds that non-oscillating data $a(x)$ generate solutions that are bounded in short time $t<2 \pi \varepsilon$. Then we consider highly-oscillating data, with characteristic frequencies $O(1 / \varepsilon)$, and show that these generate solutions that are linearly growing up to the transition time $2 \pi k_{0}$, where $k_{0}$ is the leading mode in the datum:

Theorem 1. Given $k_{0} \in \mathbb{N}^{*}$, given $0<T<2 \pi k_{0}$ and an integer $s \geq 2$, given $a \in H^{s+1}(\mathbb{T})$, if $\varepsilon$ and $|a-\bar{a}|_{H^{s+1}}$ are small enough the datum

$$
u(\varepsilon, 0, x)=a\left(\frac{k_{0} x}{\varepsilon}\right)
$$

generates a unique solution $u \in C^{0}\left([0, T], H^{s}(\mathbb{T})\right)$ to $(\mathrm{B})_{\varepsilon}$, and there holds

$$
u(\varepsilon, t, x)=i t+v\left(\varepsilon, \frac{t}{\varepsilon}, \frac{x}{\varepsilon}\right)
$$

with bounds

$$
\begin{aligned}
|\Re e v(t)-\bar{a}|_{H^{s}} & \lesssim(1+\varepsilon t C(T))\left|a\left(k_{0} \cdot\right)-\bar{a}\right|_{H^{s+1}}, \\
|\Im m v(t)|_{H^{s}} & \lesssim \varepsilon t(1+\varepsilon t C(T))\left|a\left(k_{0} \cdot\right)-\bar{a}\right|_{H^{s+1}},
\end{aligned}
$$

where $0<C(T) \rightarrow \infty$ as $T \rightarrow 2 \pi k_{0}$.

In the statement of Theorem 1, $\bar{a}$ denotes the mean mode of the initial datum: $\bar{a}:=\frac{1}{2 \pi} \int_{\mathbb{T}} a(x) d x$. The implicit multiplicative constants in 1.6 depend only on $s$ and $k_{0}$. Theorem 1 is proved in Section 3 .

Based on the numerical tests, we expect the solutions of Theorem 1 to grow exponentially for $t>2 \pi k_{0}$. This is something we can prove for small data: 
Theorem 2. Given $k_{0} \in \mathbb{N}^{*}$, given $T>0, \alpha>1 / 3$, and $a \in H^{1}(\mathbb{T})$ such that $\int_{\mathbb{T}} e^{-2 i \pi x} a(x) d x \neq 0$, if $\varepsilon$ is small enough the datum

$$
u(\varepsilon, 0, x)=\varepsilon^{\alpha} a\left(\frac{k_{0} x}{\varepsilon}\right)
$$

generates a unique solution $u \in C^{0}\left(\left[0,4 \pi k_{0}+\varepsilon T\right], H^{1}(\mathbb{T})\right)$ to $(\mathrm{B})_{\varepsilon}$, and there holds

$$
\left|\int_{\mathbb{T}} e^{-2 i \pi k_{0} x / \varepsilon} u(\varepsilon, t, x) d x\right| \geq \frac{1}{2} e^{\pi k_{0}\left(t-2 \pi k_{0}\right)^{2} / \varepsilon-4\left(\pi k_{0}\right)^{3} / \varepsilon}\left|\int_{\mathbb{T}} e^{-2 i \pi k_{0} x / \varepsilon} u(\varepsilon, 0, x) d x\right| .
$$

Theorem 2 is proved in Section 4 . The general case of $O(1)$-amplitude data seems out of reach; we explain why in Section 1.5. Note that the time-exponential lower bound in Theorem 2 starts to grow at $t=2 \pi k_{0}$, the transition time described in the introduction, and reaches the value 1 at $t=4 \pi k_{0}$, the amplification time described in the introduction. Our description of the growth stops shortly after the amplification time.

1.4. Comments. On Theorem 1 and its proof. The proof is based on the construction of an approximate solution to $(\mathrm{B})$. This procedure typically has a cost in terms of regularity. This shows in Theorem 1, as we need $a-\bar{a}$ to belong to $H^{s+1}$, while we perform estimates only in $H^{s}$. The proof uses only elementary inequalities, such as Poincaré-Wirtinger and Kato-Ponce. We note in Section 2 that existence and bounds up to the transition time are easy to derive. Our point in Theorem 1 is to obtain precise growth estimates for the imaginary part of the solution; in this view compare the crude bound (3.3) obtained at the end of our basic analysis in Section 2 with estimate (1.6).

Theorem 1 is illustrated on Figure 1 by the behavior of the numerical solutions for $N=16$ and $N=24$ before their respective amplification times, and also by Figure 3, the amplification time depends chiefly on the smallest non-zero mode in the datum. Here the smallest mode is $k_{0} / \varepsilon$, corresponding to a linear amplification time $4 \pi k_{0}$, as discussed in Section 1.2 .

This slow-then-fast dynamics is reminiscent of the phenomenon of metastability, in which a speed of convergence depends singularly on the viscosity, typically as $e^{-1 / \varepsilon}[1,7]$. These two phenomena, metastability and time-delay in the instabilities, have in common the fact that the solutions exhibit a certain stable (observable) behavior for an $O(1)$ time interval before they converge to the asymptotic limit in the case of a metastable behavior, or experience an exponential growth in time in the case of a time-delay in the instability.

On Theorem 2 and its proof. For small solutions, the approximation by the linear constant-coefficient equation (1.1) certainly makes sense at least for small time. Theorem 2 shows that qualitatively, the approximation is actually valid up to and a little bit beyond the amplification time $t=4 \pi k_{0}$, which is not clear at all at first sight.

For 1.1, the mode-by-mode analysis 1.4 shows that the Fourier modes $k / \varepsilon<0$ decay exponentially, while Fourier modes $k / \varepsilon>0$ decay until $t=2 \pi k$ (transition 
time), recover their initial value at $t=4 \pi k$ (amplification time), then grow beyond their initial value, at a rate $\sim e^{\pi t k^{2}}$, for large $t$.

The proof of Theorem 2 is based on this simple description. In particular, it is conducted entirely on the Fourier side. The smallness assumption allows to handle the nonlinear convective terms as small sources. We perform elementary estimates based on the exact description of the linear solution operator in Fourier, as seen on (1.4). The difficulty comes from error function estimates that arise in bounds for Gaussian time integrals.

1.5. Open problem. Our numerical observations lead us to conjecture that

Data with amplitude $O(1)$ and frequencies $O(1 / \varepsilon)$, of the form $a(x / \varepsilon)$, generate solutions to $(\mathrm{B})_{\varepsilon}$ which are bounded in time $O(1)$ before exhibiting an exponential growth in time.

We managed to prove the part about linear growth (Theorem 1), but we could prove exponential amplification only for small data (Theorem 2). We describe here in some detail the difficulties that arise in a sketch of proof of conjecture $(\mathrm{C})$.

Let $u(\varepsilon, 0, x)=a(x / \varepsilon)$ be given in $H^{s+1}(\mathbb{T})$. By Theorem 1, we know that the corresponding solution $u$ is defined and bounded up to time $T<2 \pi$. At $t=2 \pi$, there is a change in behavior in the associated linear constant-coefficient equation (1.1). Indeed, as we saw indirectly in (1.4), the symbol $\lambda(\varepsilon, t, \xi)=\varepsilon(2 \pi \xi)^{2}-2 \pi t \xi$ of the differential operator in (1.1) satisfies $\lambda>0$ for $t<2 \pi$ (damping) and $\lambda<0$ for $t>2 \pi$ (amplification).

Assume that $u$ is defined and bounded up to and a little bit beyond the transition time $t=2 \pi$. We posit $u=i t+\bar{a}+v$, so that $v$ solves

$$
\partial_{t} v+\frac{1}{\varepsilon}(i t+\bar{a}+v)\left(\varepsilon \partial_{x}\right) v-\frac{1}{\varepsilon}\left(\varepsilon \partial_{x}\right)^{2} v=0
$$

Since $v$ oscillates rapidly in $x$ (like its datum, presumably), it makes sense to write (1.7) in terms of $\varepsilon \partial_{x}$ derivatives, rather than $\partial_{x}$ derivatives. The symbol $\lambda(\varepsilon, t, x, \xi)$ of the differential operator in 1.7 is

$$
\lambda(\varepsilon, t, x, \xi)=(i t+\bar{a}+v(\varepsilon, t, x)) 2 i \pi \xi+(2 \pi \xi)^{2},
$$

so that (1.7) takes the form

$$
\partial_{t} v+\frac{1}{\varepsilon} \mathrm{op}_{\varepsilon}(\lambda) v=0, \quad \mathrm{op}_{\varepsilon}(\lambda) v:=\int_{\mathbb{R}^{d}} e^{2 i \pi x \cdot \xi} \lambda(\varepsilon, t, x, \varepsilon \xi) \hat{v}(\xi) d \xi .
$$

Now if locally in $(t, x, \xi)$, there holds $\Re e \lambda<0$, we expect a fast exponential growth for (1.8). The mode $\xi=1$ should be amplified first, so we will look at $\lambda$ near $\xi=1$. There holds

$$
\Re e \lambda(\varepsilon, t, x, 1)=2 \pi(2 \pi-t-\Im m v(\varepsilon, t, x)) .
$$

Since $\bar{v} \equiv 0$, we know that $\Im m v$ is not everywhere negative. In particular, for any $t>2 \pi$, we can find $x=x(t)$ such that $\Re e \lambda(\varepsilon, t, x(t), 1)<0$. We even expect $\Re e \lambda$ 
to take negative values before $t=2 \pi$, since the maximum of $\Im m v$ is nonnegative. That is, if we let

$$
t_{\star}:=\inf \left\{0<t \leq 2 \pi, \quad 2 \pi-t-\max _{\mathbb{T}} \Im m v(\varepsilon, t)<0\right\},
$$

then $t_{\star}<2 \pi$ (unless $\Im m v \equiv 0$ in a neighborhood of $t=2 \pi$, unlikely), and $t_{\star}$ appears as the transition time. At this stage, we wait a little bit longer for the transition to happen. That is, we do not handle the difficult case of a transition to ellipticity (central to [4, 8]), and instead observe the solution at some ulterior $t_{0} \in\left(t_{\star}, 2 \pi\right)$, when presumably $\Re e \lambda$ is locally negative and bounded away from zero. Let $x_{0}$ such that $\max _{\mathbb{T}} \Im m v\left(\varepsilon, t_{0}\right)=\Im m v\left(\varepsilon, t_{0}, x_{0}\right)$. We can localize around $x_{0}=0$ (by multiplication by a cut-off $\theta$ in $x$ ), then identify the solution to the truncated equation with a compactly supported function defined on $\mathbb{R}$. Then we can localize in frequency around $\xi_{0}=1$, by multiplication of the equation to the left by $\mathrm{op}_{\varepsilon}(\chi)$, where $\chi$ is a cut-off in $\xi$. The cut-off procedures produce error terms, in the form of commutators, which are smaller by a factor $\varepsilon$ since the quantization in 1.8 is semiclassical 2 Thus we arrive at

$$
\partial_{t} w+\frac{1}{\varepsilon} \operatorname{op}_{\varepsilon}(\mu) w=f
$$

where $f$ is a sum of commutators and is bounded in $\varepsilon$, and $\mu$ has the form

$$
\mu(\varepsilon, t, x, \xi)=\theta^{\sharp}(x) \chi^{\sharp}(\xi) \lambda(\varepsilon, t, x, \xi),
$$

where $\theta^{\sharp}$ is an extension of the original spatial cut-off $\theta$, and similarly $\chi^{\sharp}$ is an extension of the original frequency cut-off $\chi$. If the cut-offs are carefully chosen, then by choice of $\left(t_{0}, x_{0}\right)$, there holds $\Re e \mu \leq-\mu_{0}<0$, and the growth of $w$ follows by an application of Gårding's inequality (see for instance Theorem 4.32 in [10]). We conclude that $v$ must grow as well, and in the end that it was not reasonable to assume uniform bounds for $u$ much beyond the transition time (presumably, uniform bounds break down just after the amplification time $t=4 \pi$ ).

The difficulty that we overlooked in this discussion is the fast dependence of $v$, hence of the symbol $\lambda$, on the spatial variable $x$. In particular, we can certainly find $\left(t_{0}, x_{0}\right)$ such that $\Re e \lambda\left(\varepsilon, t_{0}, x_{0}, 1\right)<0$, but this inequality is unlikely to hold in a $O(1)$-neighborhood of $x_{0}$. Thus we would need to use an $\varepsilon$-dependent cut-off $\theta$, typically of the form $\theta\left(\left(x-x_{0}\right) / \varepsilon\right)$. Such a cut-off generates commutator that are not bounded in $\varepsilon$. Thus in 1.9 we will actually obtain a source $(1 / \varepsilon) f$, with a bounded $f$. In this setting, we would need more information on $v$, and $f$, in order to conclude by an application of Gårding's inequality.

A similar open problem (amplification proof in the presence of wildly varying coefficients) was raised in Section 1.8 of [5].

We note another difficulty, not unrelated to the small spatial scale of variation of $v$. It is that we need to have some information on $v$ at $t_{0}$ in order to prove growth.

\footnotetext{
${ }^{2}$ By composition of pseudo-differential operators in semiclassical quantization. This is already observed for differential operators, in equality $a(x) \varepsilon \partial_{x} \circ b(x) \varepsilon \partial_{x}=a(x) b(x)\left(\varepsilon \partial_{x}\right)^{2}+\varepsilon a(x) b^{\prime}(x) \varepsilon \partial_{x}$, where $\varepsilon a b^{\prime}\left(\varepsilon \partial_{x}\right)$ appears a a small remainder. For a general pseudo-differential composition result, see for instance Theorems 1.1.5 and 1.1.20 in [2].
} 
Indeed, a space-frequency truncated $v$ at $t=t_{0}$ will serve as a new initial datum for $(1.9)$ (with $(1 / \varepsilon) f$ instead of $f$ ). We will need in particular $\operatorname{op}_{\varepsilon}(\chi)(\theta v)$ not to be too small at $t=t_{0}$.

In conclusion, the difficulty here is that we are looking at the behavior of fastoscillating solutions to nonlinear equations in time $O(1)$, well after nonlinear effects have started taking place.

\section{BASIC ANALYSIS: NON-OSCILLATING DATA AND SHORT-TIME BOUNDS}

We prove here that a non-oscillating datum $u(0, x)=a(x) \in H^{s}(\mathbb{T})$ generates a unique solution $u$ up to time $T$, for any $T<2 \pi \varepsilon$, if $|a-\bar{a}|_{H^{s}}$ is small enough and $s \geq 1$. For further use we note that there holds for all $u \in H^{1}(\mathbb{T})$ the PoincaréWirtinger inequality

$$
|u-\bar{u}|_{L^{2}} \leq \frac{1}{2 \pi}\left|\partial_{x} u\right|_{L^{2}}, \quad \bar{u}:=\frac{1}{2 \pi} \int_{\mathbb{T}} u d x .
$$

We look for the solution $u$ to $(\mathrm{B})_{\varepsilon}$ in the form

$$
u(t, x)=i t+\bar{a}+v(t, x) .
$$

Then $v$ solves

$$
\partial_{t} v+(i t+\bar{a}+v) \partial_{x} v-\varepsilon \partial_{x}^{2} v=0, \quad \bar{v} \equiv 0 .
$$

Differentiating, we obtain with $v_{\alpha}:=\partial_{x}^{\alpha} v$, for $0 \leq \alpha \leq s$, with $1 \leq s$ :

$$
\frac{1}{2} \partial_{t}\left(\left|v_{\alpha}\right|_{L^{2}}^{2}\right)+\varepsilon\left|\partial_{x} v_{\alpha}\right|_{L^{2}}^{2}+\Re e\left((i t+\bar{a})\left(\partial_{x} v_{\alpha}, v_{\alpha}\right)_{L^{2}}\right)+\Re e\left(\partial_{x}^{\alpha}\left(v \partial_{x} v\right), v_{\alpha}\right)_{L^{2}}=0
$$

where the $L^{2}$ scalar product is hermitian: $(f, g)_{L^{2}}:=\int_{\mathbb{T}} \bar{f} g$. In particular, since $\bar{a} \in \mathbb{R}$, there holds $\Re e\left(\bar{a} \partial_{x} v_{\alpha}, v_{\alpha}\right)_{L^{2}}=0$, so that

$$
\left|\Re e\left((i t+\bar{a})\left(\partial_{x} v_{\alpha}, v_{\alpha}\right)_{L^{2}}\right)\right|=\left|-t \Im m\left(\partial_{x} v_{\alpha}, v_{\alpha}\right)\right| \leq t\left|\partial_{x} v_{\alpha}\right|_{L^{2}}\left|v_{\alpha}\right|_{L^{2}}
$$

Besides,

$$
\left|\Re e\left(\partial_{x}^{\alpha}\left(v \partial_{x} v\right), v_{\alpha}\right)_{L^{2}}\right| \leq \frac{1}{2}\left|v^{2}\right|_{H^{\alpha+1}}\left|v_{\alpha}\right|_{L^{2}} .
$$

By the Moser product inequality ${ }^{3}$,

$$
\left|v^{2}\right|_{H^{\alpha+1}} \leq C_{s}|v|_{L^{\infty}}|v|_{H^{\alpha+1}} .
$$

We will henceforth use $C_{s}$ as a generic notation for positive constants depending only on $s$. Then by Poincaré-Wirtinger (2.1), since $v$ has zero mean value, $|v|_{H^{\alpha+1}} \leq$ $(1+1 /(2 \pi))\left|\partial_{x} v\right|_{H^{s}}$. Gathering the above bounds, we obtain

$$
\frac{1}{2} \partial_{t}\left(\left|v_{\alpha}\right|_{L^{2}}^{2}\right)+\varepsilon\left|\partial_{x} v_{\alpha}\right|_{L^{2}}^{2} \leq t\left|\partial_{x} v_{\alpha}\right|_{L^{2}}\left|v_{\alpha}\right|_{L^{2}}+C_{s}|v|_{L^{\infty}}\left|\partial_{x} v\right|_{H^{s}}\left|v_{\alpha}\right|_{L^{2}}
$$

\footnotetext{
${ }^{3}$ In the form $\left|w_{1} w_{2}\right|_{H^{s}} \leq C_{s}\left(\left|w_{1}\right|_{L^{\infty}}\left|w_{2}\right|_{H^{s}}+\left|w_{2}\right|_{L^{\infty}}\left|w_{1}\right|_{H^{s}}\right)$, for $s \geq 0$, some $C_{s}>0$, all $w_{i} \in L^{\infty} \cap H^{s}$.
} 
Using Poincaré-Wirtinger again and then summing ${ }^{4}$ over $\alpha \leq s$, this gives

$$
\frac{1}{2} \partial_{t}\left(|v(t)|_{H^{s}}^{2}\right)+\varepsilon\left|\partial_{x} v\right|_{H^{s}}^{2} \leq\left(\frac{t}{2 \pi}+C_{s}|v|_{L^{\infty}}\right)\left|\partial_{x} v\right|_{H^{s}}^{2}
$$

In particular, so long as

$$
\frac{t}{2 \pi}+C_{s}|v|_{L^{\infty}} \leq \varepsilon
$$

there holds

$$
|v(t)|_{H^{s}} \leq|a-\bar{a}|_{H^{s}} .
$$

Thus for any $0<T<2 \pi \varepsilon$, the uniform bound (2.3) holds over all of $[0, T]$, provided that $|a-\bar{a}|_{H^{s}} \leq C_{s}(2 \pi \varepsilon-T)$. Standard arguments can be used to convert this uniform a priori estimate into an existence and uniqueness result in $L^{\infty}\left([0, T], H^{s}(\mathbb{T})\right)$, for any integer $s \geq 1$.

\section{Proof of Theorem 1; oscillating Data AND uniform Bounds in time $O(1)$}

We consider here oscillating data, of the form $u(\varepsilon, 0, x)=a\left(\frac{k_{0} x}{\varepsilon}\right)$, where $k_{0} \in \mathbb{N}$. We look for the solution $u$ to $(\mathrm{B})_{\varepsilon}$ issued from this datum in the form

$$
u(\varepsilon, t, x)=i t+\bar{a}+v\left(\frac{t}{\varepsilon}, \frac{x}{\varepsilon}\right)
$$

corresponding to a hyperbolic change of scales. Then $v$ solves

$$
\partial_{t} v+(i \varepsilon t+\bar{a}+v) \partial_{x} v-\partial_{x}^{2} v=0, \quad \bar{v} \equiv 0, \quad v(\varepsilon, 0, x)=a\left(k_{0} x\right)-\bar{a} .
$$

We observe that the datum can be expanded in Fourier series

$$
v(\varepsilon, 0, x)=\sum_{k \in \mathbb{Z}^{*}} e^{2 i \pi k \cdot x k_{0}} a_{k},
$$

where $\left(a_{k}\right)$ are the Fourier coefficients of $a$. The convective nonlinearity in (3.1) produces only harmonics of $k_{0}$. In particular, over its interval of existence, the solution $v$ to (3.1) enjoys a Fourier expansion

$$
v(\varepsilon, t, x)=\sum_{k \in \mathbb{Z}^{*}} e^{2 i \pi k \cdot x k_{0}} v_{k}(\varepsilon, t),
$$

and the Poincaré-Wirtinger inequality (2.1) takes the particular form

$$
|v|_{L^{2}} \leq \frac{1}{2 \pi k_{0}}\left|\partial_{x} v\right|_{L^{2}}
$$

\footnotetext{
${ }^{4}$ Here multiplicative constants do matter, since our goal is to reach precisely the transition time $2 \pi \varepsilon$. In particular, we define the $H^{s}$ norm as a euclidian norm: $|w|_{H^{s}}^{2}=\sum_{\alpha \leq s}\left|\partial_{x}^{\alpha} w\right|_{L^{2}}^{2}$.
} 
At this point we can reproduce the arguments of Section 2 and prove existence up to time $T<2 \pi k_{0}$. Indeed, by the same arguments as above, taking into account the scaling difference, we arrive instead of $(2.2)$ at

$$
\frac{1}{2} \partial_{t}\left(|v(t)|_{H^{s}}^{2}\right)+\left|\partial_{x} v\right|_{H^{s}}^{2} \leq\left(\frac{\varepsilon t}{2 \pi k_{0}}+C_{s}|v|_{L^{\infty}}\right)\left|\partial_{x} v\right|_{H^{s}}^{2},
$$

and the condition on $t$ now takes the form $\varepsilon t<2 \pi k_{0}$. Back in the original time scale, this gives the uniform bound

$$
\left|\left(\varepsilon \partial_{x}\right)^{\alpha} u-i t-\bar{a}\right|_{L^{2}} \leq\left|a\left(k_{0} \cdot\right)-\bar{a}\right|_{H^{s}} .
$$

Our goal in this Section is to refine this crude a priori analysis, by proving the precise growth estimate (1.6).

3.1. Approximate solution. For the solution $v$ to $(3.1)$, we posit the ansatz

$$
v=v_{1}+i \varepsilon v_{2}, \quad v_{j} \in \mathbb{R},
$$

leading to the system

$$
\left(\partial_{t}+\left(\bar{a}+v_{1}\right) \partial_{x}-\partial_{x}^{2}\right)\left(\begin{array}{l}
v_{1} \\
v_{2}
\end{array}\right)+\left(\begin{array}{cc}
0 & -\varepsilon^{2}\left(t+v_{2}\right) \\
t+v_{2} & 0
\end{array}\right) \partial_{x}\left(\begin{array}{l}
v_{1} \\
v_{2}
\end{array}\right)=0 .
$$

In a first step, we solve (3.4) for $\varepsilon=0$, corresponding to the decoupled system

$$
\left\{\begin{aligned}
\left(\partial_{t}+\left(\bar{a}+v_{1}^{a}\right) \partial_{x}-\partial_{x}^{2}\right) v_{1}^{a} & =0, \\
\left(\partial_{t}+\bar{a} \partial_{x}-\partial_{x}^{2}\right) v_{2}^{a}+\partial_{x}\left(v_{1}^{a} v_{2}^{a}\right) & =-t \partial_{x} v_{1}^{a},
\end{aligned}\right.
$$

with data

$$
v_{1}^{a}(0, x)=a\left(k_{0} x\right)-\bar{a}, \quad v_{2}^{a}(0)=0 .
$$

In this Section we derive estimates for the solution to 3.5$)-(3.6)$.

3.1.1. Estimates for $v_{1}^{a}$. There holds, by reality of $v_{1}^{a}$, for $\alpha \leq s$,

$$
\left|\Re e\left(\partial_{x}^{\alpha}\left(v_{1}^{a} \partial_{x} v_{1}^{a}\right), \partial_{x}^{\alpha} v_{1}^{a}\right)_{L^{2}}\right| \leq\left|\partial_{x} v_{1}^{a}\right|_{L^{\infty}}\left|v_{1}^{a}\right|_{H^{s}}^{2}+\left|\left[\partial_{x}^{\alpha}, v_{1}^{a}\right] \partial_{x} v_{1}^{a}\right|_{L^{2}}\left|v_{1}^{a}\right|_{H^{s}},
$$

hence, by the Kato-Ponce inequality ${ }^{5}$

$$
\left|\Re e\left(\partial_{x}^{\alpha}\left(v_{1}^{a} \partial_{x} v_{1}^{a}\right), \partial_{x}^{\alpha} v_{1}^{a}\right)_{L^{2}}\right| \leq C_{s}\left|\partial_{x} v_{1}^{a}\right|_{L^{\infty}}\left|v_{1}^{a}\right|_{H^{s}}^{2},
$$

for some $C_{s}>0$. We deduce the upper bound

$$
\frac{1}{2} \partial_{t}\left(\left|v_{1}^{a}\right|_{H^{s}}^{2}\right)+\left|\partial_{x} v_{1}^{a}\right|_{H^{s}}^{2} \leq C_{s}\left|\partial_{x} v_{1}^{a}\right|_{L^{\infty}}\left|v_{1}^{a}\right|_{H^{s}}^{2},
$$

Besides, $\partial_{x} v_{1}^{a}$ solves

$$
\left(\partial_{t}-\partial_{x}^{2}+v_{1}^{a} \partial_{x}\right)\left(\partial_{x} v_{1}^{a}\right)=-\left(\partial_{x} v_{1}^{a}\right)^{2} \leq 0,
$$

\footnotetext{
${ }^{5}$ We mean inequality $\left|\left[\partial_{x}^{\alpha}, w_{1}\right] w_{2}\right|_{L^{2}} \leq C_{s}\left(\left|\partial_{x} w_{1}\right|_{L^{\infty}}\left|w_{2}\right|_{H^{s-1}}+\left|w_{2}\right|_{L^{\infty}}\left|\partial_{x} w_{1}\right|_{H^{s-1}}\right)$, for $0 \leq \alpha \leq$ $s$, some $C_{s}>0$, all $w_{2}, \partial_{x} w_{1} \in L^{\infty} \cap H^{s-1}$.
} 
hence by the maximum principle

$$
\left|v_{1}^{a}(t)\right|_{L^{\infty}} \leq\left|a\left(k_{0} \cdot\right)-\bar{a}\right|_{L^{\infty}}, \quad\left|\partial_{x} v_{1}^{a}(t)\right|_{L^{\infty}} \leq\left|k_{0}\right|\left|\partial_{x} a\right|_{L^{\infty}} .
$$

Thus with (3.7) and Poincaré-Wirtinger, if $\left|\partial_{x} a\right|_{L^{\infty}}$ is small enough, we obtain

$$
\partial_{t}\left(\left|v_{1}^{a}\right|_{H^{s}}^{2}\right)+\left|\partial_{x} v_{1}^{a}\right|_{H^{s}}^{2} \leq 0
$$

implying the uniform bound

$$
\left|v_{1}^{a}\right|_{H^{s}} \leq\left|a\left(k_{0} \cdot\right)-\bar{a}\right|_{H^{s}},
$$

In particular, since $a$ is assumed to belong to $H^{s+1}$, we can repeat the argument in $H^{s+1}$, and obtain

$$
\left|v_{1}^{a}\right|_{H^{s+1}} \leq \boldsymbol{a}:=\left|a\left(k_{0} \cdot\right)-\bar{a}\right|_{H^{s+1}} .
$$

From (3.8) we deduce also

$$
\int_{0}^{t} \tau^{2}\left|\partial_{x} v_{1}^{a}\right|_{H^{s}}^{2} d \tau \leq-\int_{0}^{t} \tau^{2} \partial_{t}\left|v_{1}^{a}(\tau)\right|_{H^{s}}^{2} d \tau .
$$

Integrating by parts and using (3.9), this yields

$$
\int_{0}^{t} \tau^{2}\left|\partial_{x} v_{1}^{a}(\tau)\right|_{H^{s}}^{2} d \tau \leq t^{2}\left|a\left(k_{0} \cdot\right)-\bar{a}\right|_{H^{s}}^{2},
$$

corresponding to a growth of the time moment that is better than would be expected from 3.9 .

3.1.2. Estimates for $v_{2}^{a}$. We apply $\partial_{x}^{\alpha}$ to equation (3.5) (ii) in $v_{2}^{a}$ and take the scalar product with $v_{2, \alpha}^{a}:=\partial_{x}^{\alpha} v_{2}^{a}$, to obtain

$$
\frac{1}{2} \partial_{t}\left(\left|v_{2, \alpha}^{a}\right|_{L^{2}}^{2}\right)+\left|\partial_{x} v_{2, \alpha}^{a}\right|_{L^{2}}^{2} \leq\left|t\left(\partial_{x} v_{1, \alpha}^{a}, v_{2, \alpha}\right)_{L^{2}}\right|+\left|\left(\partial_{x}^{\alpha+1}\left(v_{1}^{a} v_{2}^{a}\right), v_{2, \alpha}\right)_{L^{2}}\right| .
$$

With the uniform bound (3.10), there holds

$$
\left|t\left(\partial_{x} v_{1, \alpha}^{a}, v_{2, \alpha}\right)_{L^{2}}\right| \leq t \boldsymbol{a}\left|v_{2, \alpha}^{a}\right|_{L^{2}},
$$

where notation $\boldsymbol{a}$ is introduced in (3.10), and

$$
\left|\left(\partial_{x}^{\alpha+1}\left(v_{1}^{a} v_{2}^{a}\right), v_{2, \alpha}\right)_{L^{2}}\right| \leq \boldsymbol{a}\left|v_{2}^{a}\right|_{L^{\infty}}\left|v_{2, \alpha}^{a}\right|_{L^{2}}+\left|\left[\partial_{x}^{\alpha+1}, v_{2}^{a}\right] v_{1}^{a}\right|_{L^{2}}\left|v_{2, \alpha}^{a}\right|_{L^{2}}, \quad \alpha \leq s .
$$

Using Kato-Ponce again, this gives for $\alpha \leq s$ the bound

$$
\begin{aligned}
\frac{1}{2} \partial_{t}\left(\left|v_{2, \alpha}^{a}\right|_{L^{2}}^{2}\right)+\left|\partial_{x} v_{2, \alpha}^{a}\right|_{L^{2}}^{2} & \leq t \boldsymbol{a}\left|v_{2}^{a}\right|_{H^{s}}+\boldsymbol{a}\left|v_{2}^{a}\right|_{L^{\infty}}\left|v_{2}^{a}\right|_{H^{s}} \\
& +C_{s}\left(\boldsymbol{a}\left|\partial_{x} v_{2}^{a}\right|_{L^{\infty}}\left|v_{2}\right|_{H^{s}}+|a-\bar{a}|_{L^{\infty}}\left|v_{2}^{a}\right|_{H^{s+1}}\left|v_{2}^{a}\right|_{H^{s}}\right) .
\end{aligned}
$$

If $\boldsymbol{a}$ is small enough, we can absorb the last three terms in the above upper bound by the viscous term in the left-hand side of the inequality, by use of Poincaré-Wirtinger. After summation over $0 \leq \alpha \leq s$, we are left with

$$
\partial_{t}\left(\left|v_{2}^{a}\right|_{H^{s}}^{2}\right)+\left|\partial_{x} v_{2}^{a}\right|_{H^{s}}^{2} \lesssim t\left|\partial_{x} v_{1}^{a}\right|_{H^{s}}\left|v_{2}^{a}\right|_{H^{s}}
$$


Integrating in time, we find

$$
\left|v_{2}^{a}(t)\right|_{H^{s}}^{2}+\int_{0}^{t}\left|\partial_{x} v_{2}^{a}(\tau)\right|_{H^{s}}^{2} d \tau \lesssim \int_{0}^{t} \tau^{2}\left|\partial_{x} v_{1}^{a}(\tau)\right|_{H^{s}}^{2} d \tau+\int_{0}^{t}\left|v_{2}^{a}\right|_{H^{s}}^{2},
$$

and with the uniform time-integrated bound (3.11), and another application of Poincaré-Wirtinger, we finally obtain

$$
\left|v_{2}^{a}(t)\right|_{H^{s}}^{2}+\int_{0}^{t}\left|\partial_{x} v_{2}^{a}(\tau)\right|_{H^{s}}^{2} d \tau \lesssim t^{2} \boldsymbol{a}^{2}
$$

Again, the time moment has a slower growth than would be expected from (3.13). Indeed, from (3.12), we deduce the bound

$$
\int_{0}^{t} \tau^{2}\left|\partial_{x} v_{2}^{a}\right|_{H^{s}}^{2} d \tau \lesssim \int_{0}^{t} \tau\left|v_{2}^{a}\right|_{H^{s}}^{2} d \tau+\int_{0}^{t} \tau^{3}\left|\partial_{x} v_{1}^{a}\right|_{H^{s}}\left|v_{2}^{a}\right|_{H^{s}} d \tau
$$

With 3.13 and Young's inequality this gives

$$
\int_{0}^{t} \tau^{2}\left|\partial_{x} v_{2}^{a}\right|_{H^{s}}^{2} d \tau \lesssim t^{4} \boldsymbol{a}^{2}+t^{2}\left(\int_{0}^{t} \tau^{2}\left|\partial_{x} v_{1}^{a}\right|_{H^{s}}^{2} d \tau+\int_{0}^{t}\left|v_{2}^{a}\right|_{H^{s}}^{2} d \tau\right),
$$

implying with (3.13) and the time-integrated bound (3.11) the estimate

$$
\int_{0}^{t} \tau^{2}\left|\partial_{x} v_{2}^{a}\right|_{H^{s}}^{2} d \tau \lesssim t^{4} \boldsymbol{a}^{2}
$$

3.2. The perturbation variable. We look for the solution to 3.4 in the form

$$
v_{1}=v_{1}^{a}+\varepsilon w_{1}, \quad v_{2}=v_{2}^{a}+\varepsilon w_{2},
$$

where $\left(v_{1}^{a}, v_{2}^{a}\right)$ is the approximate solution of Section 3.1. Then the perturbation variable $\left(w_{1}, w_{2}\right)$ solves

$$
\left\{\begin{aligned}
\left(\partial_{t}-\partial_{x}^{2}\right) w_{1}+\partial_{x}\left(v_{1}^{a} w_{1}\right)+\frac{\varepsilon}{2} \partial_{x}\left(w_{1}^{2}-\left(t+v_{2}^{a}+\varepsilon w_{2}\right)^{2}\right) & =0 \\
\left(\partial_{t}-\partial_{x}^{2}\right) w_{2}+\partial_{x}\left(\left(v_{1}^{a}+\varepsilon w_{1}\right) w_{2}\right) & =-\partial_{x}\left(\left(t+v_{2}^{a}\right) w_{1}\right) .
\end{aligned}\right.
$$

with null initial data: $w_{1}(0)=w_{2}(0)=0$. The equations 3.15) preserve the mean value. In particular, there holds $\bar{w}_{1}(t)=\bar{w}_{2}(t)=0$ for all $t$.

3.2.1. Estimates for $w_{1}$. There holds

$$
\frac{1}{2} \partial_{t}\left(\left|w_{1, \alpha}\right|_{L^{2}}^{2}\right)+\left|\partial_{x} w_{1, \alpha}\right|_{L^{2}}^{2}+\mathrm{I}+\varepsilon \mathrm{II}+\varepsilon \mathrm{III}=0, \quad w_{1, \alpha}:=\partial_{x}^{\alpha} w_{1}
$$

The convective terms I and II are bounded as above, integrating by parts and using Kato-Ponce. Precisely,

$|\mathrm{I}|:=\left|\Re e\left(\partial_{x}^{\alpha+1}\left(v_{1}^{a} w_{1}\right), w_{1, \alpha}\right)_{L^{2}}\right| \leq\left|\Re e\left(v_{1}^{a} \partial_{x} w_{1, \alpha}, w_{1, \alpha}\right)_{L^{2}}\right|+\left|\left[\partial_{x}^{\alpha+1}, v_{1}^{a}\right] w_{1}\right|_{L^{2}}\left|w_{1}\right|_{H^{s}}$, so that

and

$$
|\mathrm{I}| \lesssim\left|\partial_{x} v_{1}^{a}\right|_{L^{\infty}}\left|w_{1}\right|_{H^{s}}^{2}+\left|\partial_{x} v_{1}^{a}\right|_{H^{s}}\left|w_{1}\right|_{L^{\infty}}\left|w_{1}\right|_{H^{s}}
$$

$$
|\mathrm{II}|:=\frac{1}{2}\left|\Re e\left(\partial_{x}^{\alpha+1}\left(w_{1}^{2}\right), w_{1, \alpha}\right)_{L^{2}}\right| \lesssim\left|\partial_{x} w_{1}\right|_{L^{\infty}}\left|w_{1}\right|_{H^{s}}^{2} .
$$


For the third term, we simply integrate by parts so as to let $\partial_{x} w_{1, \alpha}$ appear in the $L^{2}$ scalar product. This gives

$$
\frac{\varepsilon}{2}\left|\left(\partial_{x}^{\alpha}\left(t+v_{2}\right)^{2}, w_{1, \alpha}\right)_{L^{2}}\right| \leq \varepsilon\left(t+C_{s}\left|v_{2}^{a}\right|_{H^{s}}\right)\left|v_{2}^{a}\right|_{H^{s}}\left|\partial_{x} w_{1}\right|_{H^{s}},
$$

where we used the fact that $H^{s}$ is an algebra, and

$$
\varepsilon^{3}\left|\left(\partial_{x}^{\alpha+1}\left(w_{2}^{2}\right), w_{1, \alpha}\right)_{L^{2}}\right| \leq \varepsilon^{3}\left|w_{2}^{2}\right|_{H^{s}}\left|\partial_{x} w_{1}\right|_{H^{s}},
$$

and by Moser's product inequality

$$
\varepsilon^{3}\left|\left(\partial_{x}^{\alpha+1}\left(w_{2}^{2}\right), w_{1, \alpha}\right)_{L^{2}}\right| \lesssim \varepsilon^{3}\left|w_{2}\right|_{L^{\infty}}\left|w_{2}\right|_{H^{s}}\left|\partial_{x} w_{1}\right|_{H^{s}}
$$

Finally, for the last term in III we have

$$
\varepsilon^{2}\left|\left(\partial_{x}^{\alpha+1}\left(\left(t+v_{2}^{a}\right) w_{2}\right), w_{1, \alpha}\right)_{L^{2}}\right| \leq \varepsilon^{2} t\left|w_{2}\right|_{H^{s}}\left|\partial_{x} w_{1}\right|_{H^{s}}+\varepsilon^{2} C_{s}\left|v_{2}^{a}\right|_{H^{s}}\left|w_{2}\right|_{H^{s}}\left|\partial_{x} w_{1}\right|_{H^{s}} .
$$

With the bound on $v_{1}^{a}$ from Section 3.1.1, reliant on smallness of $|a-\bar{a}|_{H^{s+1}}$, the upper bound for $\mathrm{I}$ is absorbed by the viscous term. That is, from (3.16) and the above bound on I, we find

$$
\frac{1}{2} \partial_{t}\left(\left|w_{1}\right|_{H^{s}}^{2}\right)+\left|\partial_{x} w_{1}\right|_{H^{s}}^{2} \leq C(a)\left|w_{1}\right|_{H^{s}}^{2}+\varepsilon \mathrm{II}+\varepsilon \mathrm{III},
$$

where $C(a) \rightarrow 0$ as $\boldsymbol{a}=\left|a\left(k_{0} \cdot\right)-\bar{a}\right|_{H^{s+1}} \rightarrow 0$, implying the bound

$$
\frac{1}{2} \partial_{t}\left(\left|w_{1}\right|_{H^{s}}^{2}\right)+(1-C(a))\left|\partial_{x} w_{1}\right|_{H^{s}}^{2} \leq \varepsilon \mathrm{II}+\varepsilon \mathrm{III} .
$$

We henceforth use $\sigma$ as a generic notation for positive constants which can be made arbitrarily small by choosing $\boldsymbol{a}=\left|a\left(k_{0} \cdot\right)-\bar{a}\right|_{H^{s+1}}$ small. The constant $C(a)$ above falls into this category. With the above bounds on II and III, we deduce the upper bound

$$
\frac{1}{2} \partial_{t}\left(\left|w_{1}\right|_{H^{s}}^{2}\right)+(1-\sigma)\left|\partial_{x} w_{1}\right|_{H^{s}}^{2} \leq A+\varepsilon\left(t+C_{s}\left|v_{2}^{a}\right|_{H^{s}}\right)\left|v_{2}^{a}\right|_{H^{s}}\left|\partial_{x} w_{1}\right|_{H^{s}}
$$

with notation

$$
A:=\varepsilon C_{s}\left|\partial_{x} w_{1}\right|_{L^{\infty}}\left|w_{1}\right|_{H^{s}}^{2}+\varepsilon^{2}\left(t\left(1+C_{s} \boldsymbol{a}\right)+\varepsilon C_{s}\left|w_{2}\right|_{L^{\infty}}\right)\left|w_{2}\right|_{H^{s}}\left|\partial_{x} w_{1}\right|_{H^{s}} .
$$

Using the bounds for $v_{2}^{a}$ in 3.13 , we find

$$
\int_{0}^{t} \varepsilon\left(\tau+C_{s}\left|v_{2}^{a}\right|_{H^{s}}\right)\left|v_{2}^{a}\right|_{H^{s}}\left|w_{1}\right|_{H^{s+1}} d \tau \leq \frac{C_{s}}{4 \eta} \varepsilon^{2} t^{4} \boldsymbol{a}^{2}+\eta \int_{0}^{t}\left|\partial_{x} w_{1}\right|_{H^{s}}^{2} d \tau, \quad \eta>0 .
$$

Thus from 3.17) we deduce, for $\varepsilon t \leq T$ and $0<\eta$ :

$$
\frac{1}{2}\left|w_{1}(t)\right|_{H^{s}}^{2}+(1-\sigma) \int_{0}^{t}\left|\partial_{x} w_{1}\right|_{H^{s}}^{2} \leq \frac{C_{s} T^{2}}{4 \eta} t^{2} \boldsymbol{a}^{2}+\int_{0}^{t} A d \tau .
$$

Here $\sigma$ (recall our notational convention set out just above (3.17)) depends in particular on $\eta$, the multiplicative constant coming from the elementary Young inequality. Going back to (3.17), and using (3.13), we also have

$$
(1-\sigma) \int_{0}^{t} \tau^{2}\left|\partial_{x} w_{1}\right|_{H^{s}}^{2} \leq 2 \int_{0}^{t} \tau\left|w_{1}\right|_{H^{s}}^{2} d \tau+\int_{0}^{t} \tau^{2} A d \tau+\int_{0}^{t} \varepsilon \tau^{3} C_{s}\left|v_{2}^{a}\right|_{H^{s}}\left|\partial_{x} w_{1}\right|_{H^{s}} d \tau .
$$


The last term above is in part absorbed to the left, via

$$
\int_{0}^{t} \varepsilon \tau^{3}\left|v_{2}^{a}\right|_{H^{s}}\left|\partial_{x} w_{1}\right|_{H^{s}} d \tau \leq \frac{1}{4 \eta} \int_{0}^{t} \varepsilon^{2} \tau^{4}\left|v_{2}^{a}\right|_{H^{s}}^{2} d \tau+\eta \int_{0}^{t} \tau^{2}\left|\partial_{x} w_{1}\right|_{H^{s}}^{2} d \tau
$$

which with (3.14) gives

$$
\int_{0}^{t} \varepsilon \tau^{3}\left|v_{2}^{a}\right|_{H^{s}}\left|\partial_{x} w_{1}\right|_{H^{s}} d \tau \leq \frac{C_{s} T^{2}}{4 \eta} t^{4} \boldsymbol{a}^{2}+\eta \int_{0}^{t} \tau^{2}\left|\partial_{x} w_{1}\right|_{H^{s}}^{2} d \tau .
$$

Thus we obtained the estimate

$$
(1-\sigma) \int_{0}^{t} \tau^{2}\left|\partial_{x} w_{1}\right|_{H^{s}}^{2} \leq \frac{C_{s} T^{2}}{4 \eta} t^{4} \boldsymbol{a}^{2}+2 \int_{0}^{t} \tau\left|w_{1}\right|_{H^{s}}^{2} d \tau+\int_{0}^{t} \tau^{2} A d \tau .
$$

3.2.2. Estimates for $w_{2}$. The contribution of the linear convective term $\partial_{x}\left(v_{1}^{a} w_{2}\right)$ is absorbed by the viscosity, if $\boldsymbol{a}$ (defined in (3.10) ) is small enough, just like term I in Section 3.2.1. The nonlinear convective term $\partial_{x}\left(w_{1} w_{2}\right)$ is also handled as in Section 3.2 .1

$$
\left|\left(\partial_{x}^{\alpha+1}\left(w_{1} w_{2}\right), w_{2, \alpha}\right)_{L^{2}}\right| \lesssim\left|\partial_{x} w_{1}\right|_{L^{\infty}}\left|w_{2}\right|_{H^{s}}^{2}+\left|w_{2}\right|_{L^{\infty}}\left|w_{1}\right|_{H^{s+1}}\left|w_{2}\right|_{H^{s}}
$$

Since $H^{s+1}$ is an algebra, the source term in the right-hand side of (3.15)(ii) contributes

$$
\left|\left(\partial_{x}^{\alpha+1}\left(\left(t+v_{2}^{a}\right) w_{1}\right), w_{2, \alpha}\right)_{L^{2}}\right| \leq t\left|\partial_{x} w_{1}\right|_{H^{s}}\left|w_{2}\right|_{H^{s}}+C_{s}\left|v_{2}^{a}\right|_{H^{s+1}}\left|w_{1}\right|_{H^{s+1}}\left|w_{2}\right|_{H^{s}}
$$

hence, with 3.13,

$$
\left.\left|\left(\partial_{x}^{\alpha+1}\left(\left(t+v_{2}^{a}\right) w_{1}\right), w_{2, \alpha}\right)_{L^{2}}\right| \leq t\left|\partial_{x} w_{1}\right|_{H^{s}}\left|w_{2}\right|_{H^{s}}+C_{s} \boldsymbol{a}\right)\left|w_{1}\right|_{H^{s+1}}\left|w_{2}\right|_{H^{s}}
$$

Thus we obtain

$$
\frac{1}{2} \partial_{t}\left(\left|w_{2}(t)\right|_{H^{s}}^{2}\right)+(1-C(a))\left|\partial_{x} w_{2}\right|_{H^{s}}^{2} \leq B,
$$

where $C(a) \rightarrow 0$ as $\boldsymbol{a} \rightarrow 0$, just like in Section 3.2.1. In 3.20), we introduced notation

$$
B:=\left(t+C_{s} \boldsymbol{a}\right)\left|\partial_{x} w_{1}\right|_{H^{s}}\left|w_{2}\right|_{H^{s}}+\varepsilon C_{s}\left(\left|\partial_{x} w_{1}\right|_{L^{\infty}}\left|w_{2}\right|_{H^{s}}^{2}+\left|w_{2}\right|_{L^{\infty}}\left|w_{1}\right|_{H^{s+1}}\left|w_{2}\right|_{H^{s}}\right) .
$$

3.2.3. Continuation of a priori bounds. The existence and uniqueness of the solution $\left(w_{1}, w_{2}\right)$ to 3.15 issued from $(0,0)$ is granted in very small tim 6 , with bounds

$$
\left|\partial_{x} w_{1}\right|_{L^{\infty}} \leq M_{1}(1+t), \quad\left|w_{2}\right|_{L^{\infty}} \leq M_{2}\left(1+t^{2}\right),
$$

We now show that the a priori estimates of Sections 3.2.1 and 3.2 .2 imply that bounds 3.21 are propagated up to time $T / \varepsilon$, where $T$ can be arbitrarily close to $2 \pi k_{0}$, if $|a-\bar{a}|_{H^{s+1}}$ is small enough.

So long as 3.21 holds, and $\varepsilon t \leq T$, we may bound $A$ and $B$ by 7

$$
\begin{aligned}
& A \leq C_{s} M_{1} T\left|w_{1}\right|_{H^{s}}^{2}+\varepsilon^{2} t(1+\sigma)\left|w_{2}\right|_{H^{s}}\left|\partial_{x} w_{1}\right|_{H^{s}}, \\
& B \leq C_{s} M_{1} T\left|w_{2}\right|_{H^{s}}^{2}+t(1+\sigma)\left|w_{2}\right|_{H^{s}}\left|\partial_{x} w_{1}\right|_{H^{s}} .
\end{aligned}
$$

\footnotetext{
${ }^{6}$ For instance by the arguments of Section 2

${ }^{7}$ For $t \geq 1$, corresponding to $t \geq \varepsilon$ in the original time scale, up to changing $M_{1}$ and $M_{2}$ into $M_{1} / 2$ and $M_{2} / 2$.
} 
where $\sigma=C_{s}|a-\bar{a}|_{H^{s+1}}+C_{s} M_{2} T$, consistent with the notational convention that we set out just above (3.17) if we allow $\sigma$ to depend on $M_{2}$. Plugging in (3.18) and (3.20), this gives, for $w=\left(w_{1}, w_{2}\right)$, the bound

$$
\frac{1}{2}|w|_{H^{s}}^{2}+(1-\sigma) \int_{0}^{t}\left|\partial_{x} w\right|_{H^{s}}^{2} \leq \frac{C_{s} T^{2}}{4 \eta} t^{2}|a-\bar{a}|_{H^{s+1}}^{2}+\int_{0}^{t} C_{s} M_{1} T|w|_{H^{s}}^{2} d \tau+D
$$

with notation

$$
D:=(1+\sigma) \int_{0}^{t} \tau\left|w_{2}\right|_{H^{s}}\left|\partial_{x} w_{1}\right|_{H^{s}} d \tau,
$$

where $\sigma$ depends in particular on $M_{1}$. If $M_{1}$ is small enough, depending on $T$ and $s$, we may absorb, via Poincaré-Wirtinger, the second term in the upper bound of (3.23) in the viscous term in the left-hand side, and obtain

$$
\frac{1}{2}|w|_{H^{s}}^{2}+(1-\sigma) \int_{0}^{t}\left|\partial_{x} w\right|_{H^{s}}^{2} \leq \frac{C_{s} T^{2}}{4 \eta} t^{2} \boldsymbol{a}^{2}+D
$$

We now concentrate on a bound for $D$. We are going to absorb most terms in $D$ into the viscous term in the left-hand side. To this end we will use the time-integrated bound (3.19) for $w_{1}$. By Young's inequality,

$$
(1+\sigma)^{-1} D \leq \frac{1}{4 \eta_{1}} \int_{0}^{t}\left|w_{2}\right|_{H^{s}}^{2} d \tau+\eta_{1} \int_{0}^{t} \tau^{2}\left|\partial_{x} w_{1}\right|_{H^{s}}^{2} d \tau, \quad 0<\eta_{1} .
$$

Under (3.21), by 3.22 (i), for $0<\eta_{2}$ :

$$
\begin{aligned}
\int_{0}^{t} \tau^{2} A d \tau \leq & C_{s} M_{1} \int_{0}^{t} \tau^{2}\left|w_{1}\right|_{H^{s}}^{2} d \tau+(1+\sigma) \frac{(\varepsilon t)^{2}}{4 \eta_{2}} \int_{0}^{t}\left|w_{2}\right|_{H^{s}}^{2} d \tau \\
& +(1+\sigma) \eta_{2}(\varepsilon t)^{2} \int_{0}^{t} \tau^{2}\left|\partial_{x} w_{1}\right|_{H^{s}}^{2} d \tau
\end{aligned}
$$

Using the above bound in (3.19), we obtain

$$
\left(1-\sigma-(1+\sigma) \eta_{2}(\varepsilon t)^{2}\right) \int_{0}^{t} \tau^{2}\left|\partial_{x} w_{1}\right|_{H^{s}}^{2} d \tau \leq(1+\sigma) \frac{(\varepsilon t)^{2}}{4 \eta_{2}} \int_{0}^{t}\left|w_{2}\right|_{H^{s}}^{2}+E
$$

where

$$
E:=\frac{C_{s}}{4 \eta} t^{4} \boldsymbol{a}^{2}+2 \int_{0}^{t} \tau\left|w_{1}\right|_{H^{s}}^{2} d \tau+C_{s} M_{1} \int_{0}^{t} \tau^{2}\left|w_{1}\right|_{H^{s}}^{2} .
$$

We may overlook the last term in $E$, by Poincaré-Wirtinger, up to changing $\sigma$ in (3.26). For the second term in $E$, we use 3.18):

$$
\int_{0}^{t} \tau\left|w_{1}\right|_{H^{s}}^{2} d \tau \lesssim t \int_{0}^{t}\left|\partial_{x} w_{1}\right|_{H^{s}}^{2} \lesssim \frac{C_{s}}{\eta} t^{3} \boldsymbol{a}^{2}+t \int_{0}^{t} A d \tau
$$

We use 3.22 (i) again: the contribution of the first term in $A$ is absorbed by the left-hand side, if $M_{1}$ is small enough. The contribution of the second term in $A$ has the same form as $D$, with an extra $\varepsilon^{2} t$ multiplicative prefactor. Hence we can go through the same steps, and obtain (3.26) where $E$ consists only of its first term, up to changing the values of $\sigma$. 
Back to (3.25), we obtained

$$
D \leq K \int_{0}^{t}\left|w_{2}\right|_{H^{s}}^{2}+\frac{C_{s}}{4 \eta} t^{4} \boldsymbol{a}^{2}
$$

where

$$
K:=(1+\sigma)\left(\frac{1}{4 \eta_{1}}+\eta_{1}(1+\sigma)\left(1-\sigma-\eta_{2}(\varepsilon t)^{2}\right)^{-1} \frac{(\varepsilon t)^{2}}{4 \eta_{2}}\right),
$$

which we may write, with our notational convention for $\sigma$,

$$
K=\sigma+\frac{1}{4 \eta_{1}}+\eta_{1}\left(1-\eta_{2}(\varepsilon t)^{2}\right)^{-1} \frac{(\varepsilon t)^{2}}{4 \eta_{2}} .
$$

There holds

$$
\min _{\eta_{1}>0} K=\sigma+\left(1-\eta_{2}(\varepsilon t)^{2}\right)^{-1 / 2} \frac{(\varepsilon t)}{2 \eta_{2}^{1 / 2}}
$$

By Poincaré-Wirtinger (3.2), since $w_{2}$ has zero mean and depends on $x$ through $k_{0} x$,

$$
\int_{0}^{t}\left|w_{2}\right|_{H^{s}}^{2} d \tau \leq \frac{1}{\left(2 \pi k_{0}\right)^{2}} \int_{0}^{t}\left|\partial_{x} w_{2}\right|_{H^{s}}^{2} d \tau
$$

Thus we can absorb the first term in $D$ by the left-hand side of (3.24) for $t$ and $\eta_{2}$ such that

$$
\left(1-\eta_{2}(\varepsilon t)^{2}\right)^{-1 / 2} \frac{\varepsilon t}{2 \eta_{2}^{1 / 2}}<\left(2 \pi k_{0}\right)^{2}
$$

and since

$$
\min _{\eta_{2}>0}\left(1-\eta_{2}(\varepsilon t)^{2}\right)^{-1 / 2} \frac{\varepsilon t}{2 \eta_{2}^{1 / 2}}=(\varepsilon t)^{2},
$$

we see that (3.27) amounts to $\varepsilon t<2 \pi k_{0}$, corresponding to a limiting time arbitrarily close to the transition time.

Thus we proved that in (3.24) all the terms in $D$ save for the first term in $E$ can be absorbed in the left-hand side. This gives

$$
\frac{1}{2}|w|_{H^{s}}^{2}+(1-\sigma) \int_{0}^{t}\left|\partial_{x} w\right|_{H^{s}}^{2} \leq \frac{C_{s}}{\eta} t^{4} \boldsymbol{a}^{2}
$$

under the conditions that in (3.21) the constants $M_{i}$ be small enough, and for $\eta=\eta\left(\boldsymbol{a}, T, M_{i}\right)$, where $\eta \rightarrow 0$ as $T \rightarrow 2 \pi k_{0}$. We will use (3.28) as a bound in $w_{2}$, and now go back to the bound (3.18) in $w_{1}$. By (3.22) (i), for $\sigma \leq 1$ there holds

$$
\int_{0}^{t} A \leq C_{s} M_{1} T \int_{0}^{t}\left|w_{1}\right|_{H^{s}}^{2} d \tau+\frac{\varepsilon^{2}}{\eta^{\prime}} \int_{0}^{t}\left|w_{2}\right|_{H^{s}}^{2}+\eta^{\prime}(\varepsilon t)^{2} \int_{0}^{t}\left|\partial_{x} w_{1}\right|_{H^{s}}^{2},
$$

for any $\eta^{\prime}>0$, and now using (3.28),

$$
\int_{0}^{t} A d \tau \leq\left(C_{s} M_{1} T+\frac{\eta^{\prime}(\varepsilon t)^{2}}{\left(2 \pi k_{0}\right)^{2}}\right) \int_{0}^{t}\left|\partial_{x} w_{1}\right|_{H^{s}}^{2} d \tau+\frac{C_{s}(\varepsilon T)^{2}}{\eta \eta^{\prime}} t^{4} \boldsymbol{a}^{2} .
$$

With the above bound and (3.18), we finally obtain, if $M_{1}$ and $\eta^{\prime}$ are small enough,

$$
\frac{1}{2}\left|w_{1}\right|_{H^{s}}^{2}+(1-\sigma) \int_{0}^{t}\left|\partial_{x} w_{1}\right|_{H^{s}}^{2} \leq \frac{C_{s} T^{2}}{\eta}\left(1+\frac{1}{\eta^{\prime}}\right) t^{2} \boldsymbol{a}^{2} .
$$


From $(3.28)$ and $(3.29)$, we conclude that the a priori bounds 3.21 propagate up to $T / \varepsilon$, as follows.

Given $0<\varepsilon$ and $T<2 \pi k_{0}$, the constraint $\sigma<1$ takes the form

$$
C(a)+C_{s}\left(M_{1}+M_{2}\right)+\eta+\eta^{\prime}<(1-\varepsilon)\left(1-\frac{T}{2 \pi k_{0}}\right),
$$

where $0<C(a) \rightarrow 0$ as $\boldsymbol{a} \rightarrow 0$. We first choose $M_{i}$ and $\eta, \eta^{\prime}$, such that $C_{s}\left(M_{1}+\right.$ $\left.M_{2}\right)+\eta+\eta^{\prime}$ equals one half of the upper bound in (3.30). This means in particular $\eta, \eta^{\prime} \rightarrow 0$ as $T \rightarrow 2 \pi k_{0}$. Then we choose $\boldsymbol{a}$ small enough so that not only (3.30) is satisfied, but also

$$
\boldsymbol{a} \lesssim \eta\left(M_{1}^{2}+M_{2}^{2}\right)
$$

the implicit multiplicative constant depending only on $s$ and $k_{0}$. Then $(3.28)$ and (3.29) imply that the pointwise a priori bounds (3.21) propagate up to $T / \varepsilon$.

We obtained closed a priori bounds in $C^{0}\left([0, T / \varepsilon], H^{s}\right)$. This translates into an existence and uniqueness result by classical arguments. For the original variable $u$ :

$$
u=\bar{a}+v_{1}^{a}+\varepsilon w_{1}+i\left(t+\varepsilon v_{2}^{a}+\varepsilon^{2} w_{2}\right),
$$

the bounds (3.9) and (3.13) on the approximate solution $\left(v_{1}^{a}, v_{2}^{a}\right)$ and (3.28) and (3.29) on the perturbation $\left(w_{1}, w_{2}\right)$ translate into estimates (1.6), valid for $\boldsymbol{a}=$ $\left|a\left(k_{0} \cdot\right)-\bar{a}\right|_{H^{s+1}}$ small enough, depending on $1-T /\left(2 \pi k_{0}\right)$.

\section{Proof of Theorem 2: small oscillating Data and growth in time $O(1)$}

We consider here small, highly oscillatory data $u(0, x)=\varepsilon^{\alpha} a\left(k_{0} x / \varepsilon\right)$ with $\alpha>1 / 3$ and $a \in H^{1}(\mathbb{T})$, such that $a_{1}:=\int_{\mathbb{T}} e^{-2 i \pi x} a(x) d x \neq 0$.

4.1. Uniform bounds in short time. In a first step, we prove a short-time existence result. We could simply use the result of Section 2 here, but the notation and estimates of the present Section will be useful later on. We posit the ansatz

$$
u(\varepsilon, t, x)=i t+\bar{a}+\varepsilon^{\alpha} v\left(\frac{t}{\varepsilon}, \frac{x}{\varepsilon}\right) .
$$

Then $v$ solves

$$
\partial_{t} v+\left(i \varepsilon t+\bar{a}+\varepsilon^{\alpha} v\right) \partial_{x} v-\partial_{x}^{2} v=0, \quad \bar{v} \equiv 0, \quad v(\varepsilon, 0, x)=a\left(k_{0} x\right)-\bar{a} .
$$

As in Section 3 , so long as it is defined $v(t)$ is a function of $k_{0} x$. We denote $\left(v_{k}\right)$ the Fourier mode corresponding to the $k$-th harmonics of $k_{0}$ :

$$
v(\varepsilon, t, x)=\sum_{k \in \mathbb{Z}} e^{2 i \pi k \cdot k_{0} x} v_{k}(\varepsilon, t) .
$$

There holds $v_{1}(0, x)=a_{1} \neq 0$. The leading harmonics will be $v_{1}$, in the sense that $v_{1}$ will grow from $t=2 \pi k_{0}$ on, before all other harmonics. The goal is to reach 
the amplification time $4 \pi k_{0}$, in the original time scale. In the time scale for $v$, this corresponds to $4 \pi k_{0} / \varepsilon$. We let

$$
w(t, x):=v(t, x)-e^{i x} v_{1}(t)=\sum_{k \notin\{0,1\}} e^{2 i \pi k \cdot k_{0} x} v_{k}(t)=: \sum_{k \in \mathbb{Z}} e^{2 i \pi k \cdot k_{0} x} w_{k}(t) .
$$

Then, $v_{1}$ and $w$ solve the triangular system

$$
\left\{\begin{aligned}
\partial_{t} v_{1}+2 \pi k_{0}\left(i \bar{a}+2 \pi k_{0}-\varepsilon t\right) v_{1} & =\varepsilon^{\alpha} f(w, w), \\
\partial_{t} w+(i \varepsilon t+\bar{a}) \partial_{x} w-\partial_{x}^{2} w & =\varepsilon^{\alpha} \partial_{x} g(v, v),
\end{aligned}\right.
$$

with notation

$$
f(w, w):=-2 i \pi k_{0} \sum_{k_{1}+k_{2}=1} w_{k_{1}} w_{k_{2}}, \quad g(v, v):=-\sum_{k \neq 1} e^{2 i \pi k_{0} \cdot k x} \sum_{k_{1}+k_{2}=k} v_{k_{1}} v_{k_{2}} .
$$

In integral form,

$$
\begin{aligned}
& v_{1}(t)=e^{\lambda_{1}(t)} v_{1}(0)+\varepsilon^{\alpha} \int_{0}^{t} e^{\lambda_{1}(t)-\lambda_{1}(\tau)} f(w, w)(\tau) d \tau \\
& w_{k}(t)=e^{\lambda_{k}(t)} w_{k}(0)+\varepsilon^{\alpha}\left(2 i \pi k_{0}\right) k \int_{0}^{t} e^{\lambda_{k}(t)-\lambda_{k}(\tau)} \widehat{g(v, v)}(\tau, k) d \tau .
\end{aligned}
$$

where $\lambda_{k}(t)$ stands for

$$
\lambda_{k}(t):=-2 i \pi k_{0} k \bar{a} t+\pi k_{0} k\left(\varepsilon t^{2}-4 \pi k_{0} k t\right) .
$$

There holds, by Young's convolution inequality and Parseval's equality, the pointwise bounds

$$
\left|f\left(z_{1}, z_{2}\right)\right| \leq\left|z_{1}\right|_{L^{2}}\left|z_{2}\right|_{L^{2}}, \quad\left|g\left(\widehat{z_{1}, z_{2}}\right)\right| \leq\left|z_{1}\right|_{L^{2}}\left|z_{2}\right|_{L^{2}} .
$$

For $0 \leq t$ and $k \leq-1$, we will use the elementary bounds

$$
e^{\Re e \lambda_{k}(t)} \leq e^{-t\left(2 \pi k_{0} k\right)^{2}}, \quad \int_{0}^{t} e^{\Re e \lambda_{k}(t)-\Re e \lambda_{k}(\tau)}|k| d \tau \leq \int_{0}^{t} e^{-(t-\tau)\left(2 \pi k_{0} k\right)^{2}}|k| d \tau \lesssim \frac{1}{|k|} .
$$

For $3 \leq k$ and $0 \leq \tau \leq t \leq \frac{5 \pi k_{0}}{\varepsilon}$,

$$
\Re e \lambda_{k}(t)=-t\left(2 \pi k_{0}\right)^{2} k(k-5 / 4),
$$

and

$$
\begin{aligned}
\Re e \lambda_{k}(t)-\Re e \lambda_{k}(\tau) & =(t-\tau) \pi k_{0} k\left(\varepsilon(t+\tau)-4 \pi k_{0} k\right) \\
& \leq-(t-\tau)\left(2 \pi k_{0}\right)^{2} k(k-5 / 2) .
\end{aligned}
$$

We deduce the a priori bound, for $0 \leq t \leq 5 \pi k_{0} / \varepsilon$, where $5 \pi k_{0} / \varepsilon$ is only a convenient limiting time that is greater than our target $4 \pi k_{0} / \varepsilon$ :

$$
\left|w_{k}(t)\right| \lesssim e^{-t\left(2 \pi k_{0}\right)^{2}|k|}\left|w_{k}(0)\right|+\frac{\varepsilon^{\alpha}}{|k|}|v|_{L^{\infty}\left([0, t], L^{2}(\mathbb{T})\right)}^{2}, \quad k \leq-1 \text { and } 3 \leq k .
$$


From the equality in 4.5 , we see that we cannot obtain a good decay estimate for the Duhamel term in $w_{2}$ up to $t=4 \pi k_{0} / \varepsilon$. For $0 \leq \tau \leq t \leq 2 \pi k_{0} / \varepsilon$,

$$
\left|w_{2}(t)\right| \lesssim e^{-t\left(2 \pi k_{0}\right)^{2} k}\left|w_{2}(0)\right|+\varepsilon^{\alpha}|v|_{L^{\infty}\left([0, t], L^{2}(\mathbb{T})\right)}^{2} .
$$

By summation in $\ell_{2}$, the bounds (4.6) and (4.7) give

$$
|w(t)|_{L^{2}} \lesssim e^{-t\left(2 \pi k_{0}\right)^{2}}\left|a\left(k_{0} \cdot\right)-\bar{a}\right|_{L^{2}}+\varepsilon^{\alpha}|v|_{L^{\infty}\left([0, t], L^{2}(\mathbb{T})\right)}^{2} \cdot
$$

In order to bound the equation in $v_{1}$, we compute

$$
\int_{0}^{t} e^{\Re e \lambda_{1}(t)-\Re e \lambda_{1}(\tau)} d \tau=e^{\pi k_{0} \varepsilon\left(t-2 \pi k_{0} / \varepsilon\right)^{2}} \int_{0}^{t} e^{-\pi k_{0} \varepsilon\left(\tau-2 \pi k_{0} / \varepsilon\right)^{2}} d \tau .
$$

This last error function integral can be evaluated for $t<2 \pi k_{0} / \varepsilon$ : indeed we have then

$$
\begin{aligned}
\int_{0}^{t} e^{-\pi k_{0} \varepsilon\left(\tau-2 \pi k_{0} / \varepsilon\right)^{2}} d \tau & =\left(\pi k_{0} \varepsilon\right)^{-1 / 2} \int_{\left(\pi k_{0} \varepsilon\right)^{1 / 2}\left(2 \pi k_{0} / \varepsilon-t\right)}^{2\left(\pi k_{0}\right)^{3 / 2} \varepsilon^{-1 / 2}} e^{-z^{2}} d z \\
& \leq\left(\pi k_{0} \varepsilon\right)^{-1 / 2} \int_{\left(\pi k_{0} \varepsilon\right)^{1 / 2}\left(2 \pi k_{0} / \varepsilon-t\right)}^{\infty} e^{-z^{2}} d \zeta \\
& \leq \frac{1}{2 \pi k_{0}\left(2 \pi k_{0}-\varepsilon t\right)} e^{-\pi k_{0} \varepsilon\left(t-2 \pi k_{0} / \varepsilon\right)^{2}}
\end{aligned}
$$

where in the last inequality we used

$$
\int_{x}^{\infty} e^{-z^{2}} d z \leq \frac{e^{-x^{2}}}{2 x}, \quad 0<x .
$$

This gives, for $0 \leq t<2 \pi k_{0} / \varepsilon$ :

$$
\left|v_{1}(t)\right| \lesssim\left|v_{1}(0)\right|+\frac{\varepsilon^{\alpha}}{2 \pi k_{0}-\varepsilon t}|w|_{L^{\infty}\left([0, t], L^{2}(\mathbb{T})\right)}^{2} .
$$

The a priori bounds, (4.8) and 4.10), are uniform with respect to $\varepsilon$ over times intervals $[0, T(\varepsilon) / \varepsilon]$, with $T(\varepsilon)=2 \pi k_{0}-O\left(\varepsilon^{\alpha}\right)$.

4.2. Beyond the transition time $t=2 \pi k_{0}$. In the estimates of the previous Section, the main error came from the error function estimate 4.9 associated with the propagator in the equation in $v_{1}$. In the current Section, we factor out this propagator. This allows for sharper estimates. We let, using notation $\lambda_{1}$ introduced in 4.3 ,

$\tilde{v}_{1}(t):=e^{-\Re e \lambda_{1}(t)} v_{1}(t), \quad \tilde{w}_{k}(t):=e^{-\Re e \lambda_{1}(t)} w_{k}(t), \quad \tilde{w}(t, x):=\sum_{k \notin\{0,1\}} e^{2 i \pi k_{0} \cdot k x} \tilde{w}_{k}(t)$.

We also denote $\tilde{v}(t):=e^{-\Re e \lambda_{1}(t)} v(t)$, with Fourier coefficients $\tilde{v}_{k}$ (equal to $\tilde{w}_{k}$ if $k \neq 1$, to $\tilde{v}_{1}$ otherwise). Then, $\tilde{v}_{1}$ and $\tilde{w}_{k}$ solve

$$
\begin{aligned}
& \tilde{v}_{1}(t)=v_{1}(0)+\varepsilon^{\alpha} \int_{0}^{t} f(\tilde{w}, w)(\tau) d \tau \\
& \tilde{w}_{k}(t)=e^{\mu_{k}(t)} w_{k}(0)+\varepsilon^{\alpha}\left(2 i \pi k_{0}\right) k \int_{0}^{t} e^{\mu_{k}(t)-\mu_{k}(\tau)} \widehat{g(\tilde{v}, v)}(\tau, k) d \tau,
\end{aligned}
$$


with notation

$$
\mu_{k}(t):=\lambda_{k}(t)-\Re e \lambda_{1}(t)=-2 i \pi k_{0} k \bar{a} t+\pi k_{0}(k-1) \varepsilon t^{2}-4\left(\pi k_{0}\right)^{2}\left(k^{2}-1\right) t,
$$

so that

$$
\Re e \mu_{k}(t)=\pi k_{0}(k-1) t\left(\varepsilon t-4 \pi k_{0}(k+1)\right) .
$$

Let $M \geq 2$ and $t_{\star}(\varepsilon, M)$ such that

$$
|v(t)| \leq M\left|a\left(k_{0} \cdot\right)-\bar{a}\right|_{L^{2}} .
$$

uniformly in $t \in\left[0, t_{\star}(\varepsilon)\right]$ and in $\varepsilon \in\left(0, \varepsilon_{0}\right)$, for $\varepsilon_{0}$ small enough (depending on $M)$, where $t_{\star}(\varepsilon, M)$ is a final observation time, smaller than $5 \pi k_{0} / \varepsilon$. We denote $t_{\star}(\varepsilon)=t_{\star}(\varepsilon, M)$ in the following. The analysis of the previous Section implies that such a bound exists if $t_{\star}(\varepsilon)=T(\varepsilon) / \varepsilon=2 \pi k_{0} / \varepsilon-O\left(\varepsilon^{\alpha-1}\right)$. Our goal here is to extend this limiting time.

4.2.1. Large Fourier indices. For $k \leq-2$, the corresponding modes are decaying, since $\Re e \mu_{k}(t)$ is negative and decaying for all $t>0$. Here we can simply overlook the contribution of $i t \partial_{x}$ to $\Re e \mu_{k}$, and obtain, for $t \leq t_{\star}(\varepsilon)$ :

$$
\left|\tilde{w}_{k}(t)\right| \lesssim e^{-4 \pi k_{0} t\left(k^{2}-1\right)}\left|w_{k}(0)\right|+\frac{\varepsilon^{\alpha} M}{|k|}|\tilde{v}|_{L^{\infty} L^{2}}, \quad k \leq-2 .
$$

The modes associated with $k \geq 2$ are not growing in our observation window $\left[0,5 \pi k_{0} / \varepsilon\right]$. Indeed, for $0 \leq \tau \leq t \leq 5 \pi k_{0} / \varepsilon$ and $2 \leq k$, there holds, by 4.11,

$$
\Re e \mu_{k}(t)=t(k-1)\left(\frac{\varepsilon t}{2}-(k+1)\right) \leq-\left(2 \pi k_{0}\right)^{2} t\left(k^{2}-1 / 4\right),
$$

and

$$
\begin{aligned}
\Re e \mu_{k}(t)-\Re e \mu_{k}(\tau) & =\pi k_{0}(t-\tau)(k-1)\left(\varepsilon(t+\tau)-4 \pi k_{0}(k+1)\right) \\
& \leq-\left(2 \pi k_{0}\right)^{2}(t-\tau)\left(k^{2}-3 / 2\right) .
\end{aligned}
$$

Thus for $t \leq t_{\star}(\varepsilon)$ :

$$
\left|\tilde{w}_{k}(t)\right| \lesssim e^{-\left(2 \pi k_{0}\right)^{2} t\left(k^{2}-1 / 4\right)}\left|w_{k}(0)\right|+\frac{\varepsilon^{\alpha} M}{|k|}|\tilde{v}|_{L^{\infty} L^{2}}, \quad 2 \leq k .
$$

4.2.2. Small Fourier indices. These are $k=-1$ and $k=1$. For $k=-1$, the diffusion fails to provide decay. We could simply use the convolution bound (4.4) and obtain

$$
\left|\tilde{w}_{-1}(t)\right| \lesssim e^{-2 \pi k_{0} \varepsilon t^{2}}\left|w_{-1}(0)\right|+\varepsilon^{\alpha} M|\tilde{v}|_{L^{\infty} L^{2}} \int_{0}^{t} e^{-2 \pi k_{0} \varepsilon\left(t^{2}-\tau^{2}\right)} d \tau
$$

but this would bring out a factor $\varepsilon^{\alpha-1 / 2}$, as in (4.17) below, and impose condition $\alpha>1 / 2$. The bound (4.15) can be refined as follows.

We observe that, since the mean mode is identically zero, all the terms in the sum $\widehat{g(\tilde{v}, v)}(t,-1)$ have the form $\tilde{w}_{k_{1}} v_{k_{2}}$, with $k_{1} \leq-2$. For $v_{k_{2}}$ we use the postulated 
bound (4.12). For $\tilde{w}_{k_{1}}$ we use the decaying bound $(4.13)$, so that the contribution of $\tilde{w}_{k_{1}} v_{k_{2}}$ to the Duhamel term in $\tilde{w}_{-1}$ is controlled by

$$
\varepsilon^{\alpha} M \int_{0}^{t} e^{-2 \pi k_{0} \varepsilon\left(t^{2}-\tau^{2}\right)}\left(e^{-4 \pi k_{0} \tau\left(k_{1}^{2}-1\right)}\left|w_{k_{1}}(0)\right|+\frac{\varepsilon^{\alpha} M}{\left|k_{1}\right|}|\tilde{v}|_{L^{\infty} L^{2}}\right) d \tau,
$$

up to a multiplicative constant, for $t \leq t_{\star}(\varepsilon)$, with $k_{1} \leq-2$. The first term above is controlled by $\varepsilon^{\alpha} M k_{1}^{-2}\left|w_{k_{1}}(0)\right|_{L^{2}}$. For the second term in (4.16), we use the elementary bound

$$
\begin{aligned}
\int_{0}^{t} e^{-2 \pi k_{0} \varepsilon\left(t^{2}-\tau^{2}\right)} d \tau & =e^{-2 \pi k_{0} \varepsilon t^{2}}\left(2 \pi k_{0} \varepsilon\right)^{-1 / 2} \int_{0}^{\left(2 \pi k_{0} \varepsilon\right)^{1 / 2} t} e^{z^{2}} d z \\
& \leq e^{-2 \pi k_{0} \varepsilon t^{2}}\left(2 \pi k_{0} \varepsilon\right)^{-1 / 2}\left(\int_{0}^{1} e^{z^{2}} d z+\int_{1}^{\left(2 \pi k_{0} \varepsilon\right)^{1 / 2} t} z e^{z^{2}} d z\right) \lesssim \varepsilon^{-1 / 2}
\end{aligned}
$$

Summing over $k_{1}$, we obtain

$$
\left|\tilde{w}_{-1}(t)\right| \lesssim e^{-\left(2 \pi k_{0}\right) \varepsilon t^{2}}\left|w_{-1}(0)\right|+\varepsilon^{\alpha} M|v(0)|_{L^{2}}+\varepsilon^{2 \alpha-1 / 2} M^{2}|\tilde{v}|_{L^{\infty} L^{2}}
$$

We turn to $k=1$. All the terms in $f(\tilde{w}, w)$ have the form $\tilde{w}_{k_{1}} w_{k_{2}}$, where $k_{1} \geq 2$ and $k_{2} \leq-1$. For $\tilde{w}_{k_{1}}$ we use (4.14). For $w_{k_{2}}$ we use (4.6), and obtain a control by

$$
\begin{aligned}
\varepsilon^{\alpha} \int_{0}^{t}\left(e^{-\left(2 \pi k_{0}\right)^{2} \tau\left(k_{1}^{2}-1 / 4\right)}\left|w_{k_{1}}(0)\right|\right. & \left.+\frac{\varepsilon^{\alpha} M}{\left|k_{1}\right|}|\tilde{v}|_{L^{\infty} L^{2}}\right) \\
& \times\left(e^{-\left(2 \pi k_{0}\right)^{2} \tau\left|k_{2}\right|}\left|w_{k_{2}}(0)\right|+\frac{\varepsilon^{\alpha} M^{2}}{\left|k_{2}\right|}\right) d \tau .
\end{aligned}
$$

Bounding from above the time integrals and summing over $k_{1}$, we find that the above term is controlled by

$$
\varepsilon^{\alpha}|v(0)|_{L^{2}}^{2}\left(1+\varepsilon^{\alpha}|\tilde{v}|_{L^{\infty} L^{2}}\right)+t \varepsilon^{3 \alpha} M^{3}|\tilde{v}|_{L^{\infty} L^{2}} .
$$

This implies for $\tilde{v}_{1}$ the bound, for $t \leq t_{\star}(\varepsilon)$,

$$
\left|\tilde{v}_{1}(t)\right| \lesssim\left|v_{1}(0)\right|+\varepsilon^{\alpha}|v(0)|_{L^{2}}^{2}\left(1+\varepsilon^{\alpha}|\tilde{v}|_{L^{\infty} L^{2}}\right)+t \varepsilon^{3 \alpha} M^{3}|\tilde{v}|_{L^{\infty} L^{2}}
$$

4.2.3. Continuation of a priori bounds. We now gather 4.13, 4.14, 4.18) and (4.20). Summing over $k$ and taking into account the definition of $\tilde{v}$ at the beginning of Section 4.2, we obtain for $\varepsilon$ small enough (depending on $M$ ) the a priori bound

$$
|v(t)|_{L^{2}} \leq 2 e^{\Re e \lambda_{1}(t)}|v(0)|_{L^{2}}, \quad t \leq \min t_{\star}(\varepsilon),
$$

By definition,

$$
\Re e \lambda_{1}(t)=\varepsilon \pi k_{0}\left(t-\frac{2 \pi k_{0}}{\varepsilon}\right)^{2}-\frac{4\left(\pi k_{0}\right)^{3}}{\varepsilon} .
$$

We now let $t_{\star}(\varepsilon)=\frac{4 \pi k_{0}}{\varepsilon}+T_{\star}$. So long as

$$
\left(2 \pi k_{0}\right)^{2} T_{\star}+\varepsilon \pi k_{0} T^{2} \leq \ln \left(\frac{M}{2}\right)
$$


we see from (4.21) that the a priori bound $(4.12$ propagates beyond the transition time $t=2 \pi k_{0} / \varepsilon$, beyond the amplification time $t=4 \pi k_{0} / \varepsilon$, and up to $t_{\star}(\varepsilon)$ as defined above.

4.3. Amplification. Integrating the equation in $\tilde{v}_{1}$ over $[0, t]$, with $t \leq t_{\star}(\varepsilon)$, the observation time $t_{\star}(\varepsilon)$ being defined just above, we find by the triangular inequality the lower bound

$$
\left|\tilde{v}_{1}(t)\right| \geq\left|v_{1}(0)\right|-\varepsilon^{\alpha} \int_{0}^{t_{\star}(\varepsilon)}|f(\tilde{w}, w)(\tau)| d \tau .
$$

We can bound the above Duhamel term with 4.19, and this gives

$$
\left|\tilde{v}_{1}(t)\right| \geq\left|v_{1}(0)\right|-\varepsilon^{\alpha}|v(0)|_{L^{2}}^{2}\left(1+\varepsilon^{\alpha}|\tilde{v}|_{L^{\infty} L^{2}}\right)+\varepsilon^{3 \alpha-1} M^{3}|\tilde{v}|_{L^{\infty} L^{2}}
$$

In particular, for $\varepsilon$ small enough,

$$
\left|\tilde{v}_{1}(t)\right| \geq \frac{1}{2}\left|v_{1}(0)\right|
$$

Back in the original variable, this gives the exponential lower bound

$$
\left|v_{1}(t)\right| \geq \frac{1}{2} e^{\Re e \lambda_{1}(t)}\left|v_{1}(0)\right|=\frac{1}{2} e^{\varepsilon \pi k_{0}\left(t-2 \pi k_{0} \varepsilon\right)^{2}-4\left(\pi k_{0}\right)^{3} / \varepsilon}\left|v_{1}(0)\right|,
$$

as claimed in the statement of Theorem 2, Indeed, by definition of $v$ in (4.1) and $v_{1}$ in (4.2), there holds $v_{1}(t)=\varepsilon^{-1} \int_{\mathbb{T}} e^{-2 i \pi k_{0} x / \varepsilon} u(\varepsilon t, x) d x$. In particular, evaluating at $t=t_{\star}(\varepsilon)$, where the final observation time is defined in Section 4.2 .3 above, we see that if $M$ is large enough (implying $\varepsilon$ small enough), then $\left|v_{1}\left(t_{\star}(\varepsilon)\right)\right| \geq 4|v(0)|_{L^{\infty}}$, implying if $\bar{a}=0$ the lower bound $\left|u\left(\varepsilon t_{\star}(\varepsilon)\right)\right|_{L^{\infty}} \geq 4|u(0)|_{L^{\infty}}$, as in the final time-step of our simulations.

\section{REFERENCES}

[1] G. Kreiss, H. Kreiss, Convergence to steady state of solutions of Burgers' equation, Appl. Numer. Math. 2 (1986), no. 3-5, 161-179.

[2] N. Lerner, Metrics on the Phase Space and Non-Selfadjoint Pseudodifferential Operators, Pseudo-Differential Operators. Theory and Applications, 3. Birkhäuser 2010. xii+397 pp.

[3] N. Lerner, Y. Morimoto, C. J. Xu, Instability of the Cauchy-Kovalevskaya solution for a class of non-linear systems, American J. Math., 132 (2010), no 1, 99-123.

[4] N. Lerner, T. Nguyen, B. Texier, The onset of instability in first-order systems, arXiv: 1504.04477.

[5] L. Yong, B. Texier, A stability criterion for high-frequency oscillations, arXiv:1307.4196, to appear in Mém. Soc. Math. Fr.

[6] G. Métivier, Remarks on the well-posedness of the nonlinear Cauchy problem, Geometric analysis of PDE and several complex variables, Contemp. Math., vol. 368, Amer. Math. Soc., Providence, RI, 2005, 337-356.

[7] C. Mascia, M. Strani, Metastability for nonlinear parabolic equations with applications to viscous scalar conservation laws, SIAM J. Math. Anal. 45 (2013), no 5, 3084-3113.

[8] B. Morisse, On hyperbolicity and well-posedness in Gevrey spaces, in preparation. 
[9] B. Texier, Approximations of pseudo-differential flows, arXiv:1402.6868, to appear in Indiana Univ. Math. J.

[10] M. Zworski, Semiclassical analysis, Graduate Studies in Mathematics, 138. American Mathematical Society, 2012. xii+431 pp.

Dipartimento di Matematica e Applicazioni, Università di Milano Bicocca

E-mail address: marta.strani@unimib.it

Institut de Mathématiques de Jussieu-Paris Rive Gauche UMR CNRS 7586, UniverSITÉ PARIS-DIDEROT

E-mail address: benjamin.texier@imj-prg.fr 